\title{
Zu dieser Ausgabe
}

In diesem Jahr begehen wir den 100. Geburtstag des bedeutenden marxistisch-leninistischen Wissenschaftlers Eugen Varga (1879-1964). Es kann wohl kaum eine bessere Form der Würdigung seines Lebenswerks in unserer Deutschen Demokratischen Republik geben, als seine Schriften in einer möglichst repräsentativen Auswahl neu herauszugeben. Eugen Vargas Werk hat bis heute seine Aktualität für die politökonomische Forschung und Lehre sowie für die gesamte ideologische Auseinandersetzung mit dem Imperialismus bewahrt. Seine schöpferischen Leistungen als Politökonom wie sein leidenschaftliches Eintreten für den revolutionären Kampf der Arbeiterklasse waren ein Vorbild für Generationen kommunistischer Gesellschaftswissenschaftler in vielen Ländern der Erde.

Eugen Varga wurde 1879 in Ungarn geboren. Seit seiner Jugend war er mit der Arbeiterbewegung verbunden und gehörte zum linken Flügel der Sozialdemokratie. In den Jahren vor dem ersten Weltkrieg veröffentlichte Varga Beiträge im theoretischen Organ der deutschen Sozialdemokratie „Neue Zeit“ vor allem über die ökonomische Entwicklung und die Lage der Arbeiterklasse in Ungarn sowie über Probleme der Geldtheorie. Einen Höhepunkt im Leben des Revolutionärs und Wissenschaftlers bildete seine Tätigkeit während der Ungarischen Räterepublik im Jahre 1919. In den Funktionen eines Volkskommissars und später des Vorsitzenden des Obersten Volkswirtschaftsrates gehörte Eugen Varga zu den führenden Persönlichkeiten der Ungarischen Räterepublik. In dieser Periode des schärfsten Klassenkampfes setzte er sich mit ganzer Kraft für sozialistische Umgestaltungen der durch den Krieg und die konterrevolutionäre Intervention zerrütteten Wirtschaft Ungarns ein. In seiner berühmten Schrift „Die wirtschaftspolitischen Probleme der proletarischen Diktatur" hat Eugen Varga unmittelbar nach der Niederlage der sozialistischen Revolution in Ungarn diese Tätigkeit kritisch eingeschätzt, die gewonnenen Erfahrungen zu verallgemeinern gesucht, um sie für den weiteren Kampf der internationalen Arbeiterklasse nutzbar zu machen.

Nach dem Zusammenbruch der Ungarischen Räterepublik entkam Eugen Varga zunächst nach Österreich und siedelte 1920 in die Sowjetunion über. Hier, in dem ersten sozialistischen Staat, in dem Bollwerk des revolutionären Kampfes der internationalen Arbeiterklasse, fand er seine zweite Heimat. Es begann eine neue wichtige Periode im politischen und wissenschaftlichen Leben Eugen Vargas. Auf Empfehlung der Kommunistischen Internationale übernahm der die verantwortungsvolle Aufgabe, regelmäßig Analysen über die Entwicklung der Weltwirtschaft und über die Wirtschaftspolitik in den kapitalistischen Ländern anzufertigen. Diese Tätigkeit, die auch mit einer Funktion eines Wirtschaftsberaters der Kommunistischen Internationale verbunden war, fand ihren Niederschlag vor allem in den Vierteljahresberichten zur Wirtschaft und Wirtschaftspolitik (1922-1939) sowie in zahlreichen Schriften über die Nachkriegskrise und die Niedergangsperiode im Kapitalismus nach dem ersten Weltkrieg. Im Jahre 1927 übernahm Eugen Varga die Leitung des neugegründeten ,Instituts für Weltwirtschaft und Welt- 
politik", das bis zum Jahre 1947 bestand. Jahrelang wirkte Eugen Varga auch als Herausgeber der gleichnamigen Zeitschrift in der UdSSR. Gerade die Zeitspanne zwischen den beiden Weltkriegen ist durch ein außerordentlich umfangreiches und fruchtbares Schaffen Eugen Vargas auf dem Gebiet der politischen Ökonomie des Kapitalismus gekennzeichnet. In dieser Zeit entstanden seine berühmten Arbeiten über die „Große Krise“ 1929-1933, über die Depression besonderer Art und die zyklische Bewegung der kapitalistischen Wirtschaft am Vorabend des zweiten Weltkrieges. Zugleich beschäftigte sich Eugen Varga eingehend mit den sozialen Prozessen in den Hauptländern des Kapitals. Besonders hervorzuheben ist sein grundlegender Beitrag zur Ausarbeitung einer Theorie der allgemeinen Krise des Kapitalismus. In diesen Jahren wurden Eugen Varga auch zahlreiche politische Aufgaben durch die Kommunistische Internationale und die Sowjetregierung übertragen. So wirkte er u. a. von 1922 bis 1927 als wissenschaftlicher Berater der Handelsvertretung Sowjetrußlands in Deutschland.

Gleichzeitig übte Eugen Varga eine umfangreiche Lehrtätigkeit aus. Er erzog eine ganze Generation marxistisch-leninistischer Gesellschaftswissenschaftler, die in vielen kommunistischen Parteien tätig waren. So spielte er eine maßgebliche Rolle an dem von ihm geleiteten Institut der Roten Professur für Weltwirtschaft und Weltpolitik, das in den 30er Jahren bestand. Im Jahre 1939 wurde er zum Ordentlichen Mitglied der Akademie der Wissenschaften der UdSSR gewählt und gehörte lange Zeit dem Präsidium dieser Akademie an. In dieser Tätigkeit erwies sich Eugen Varga als ein umfassend gebildeter kenntnisreicher Wissenschaftler, der durch viele anregende Beiträge auch in wissenschaftlichen Diskussionen über naturwissenschaftliche und technische Probleme auftrat.

In der Zeit des zweiten Weltkrieges erschienen aus seiner Feder zahlreiche Schriften über die Entwicklung der Wirtschaft und Politik in den kriegführenden Staaten. Er beschäftigte sich besonders mit der Entwicklung der Kriegswirtschaft und der inneren politischen Lage im faschistischen Deutschland. Als Zusammenfassung seiner einzelnen Studien und als Verallgemeinerung seiner Forschung über die Entwicklungstendenzen des Kapitalismus erschien 1946 sein Buch „,Veränderungen in der Wirtschaft des Kapitalismus im Ergebnis des zweiten Weltkrieges“, das die bekannte „Varga-Diskussion“ auslöste.

Als 1956 das neue „Institut für Weltwirtschaft und Internationale Beziehungen“ an der Akademie der Wissenschaften der UdSSR gegründet wurde, gehörte Eugen Varga zu den Begründern und aktiven Beratern dieser neuen Einrichtung, die seitdem eine bedeutende, international anerkannte wissenschaftliche Tätigkeit ausübt.

Eugen Varga hat sich auch nach dem zweiten Weltkrieg bis zu seinem Tode im Jahre 1964 weiter mit den grundlegenden Entwicklungstendenzen des Kapitalismus beschäftigt. Als Ergebnis dieser Untersuchungen entstanden zahlreiche Artikel und Schriften, in denen Eugen Varga den Versuch unternahm, die Veränderungen in der zyklischen Bewegung der kapitalistischen Wirtschaft und in den langfristigen Entwicklungstendenzen des Kapitalismus unter den Bedingungen der Vertiefung der allgemeinen Krise zu untersuchen. Seine bekannteste Schrift aus dieser letzten Schaffensperiode ist die Arbeit „Der Kapitalismus des zwanzigsten Jahrhunderts“.

Auch wenn die Schilderung des Lebensweges Eugen Vargas im Rahmen dieses Vorwortes nur sehr knapp ausfallen kann, so gibt sie doch eine Vorstellung von dem Revolutionär und Wissenschaftler Eugen Varga. Eine Würdigung dieser hervorragenden Persönlichkeit bliebe aber unvollständig, würde man nicht wenigstens den Versuch unter- 
nehmen, seine persönlichen Eigenschaften und seine Arbeitsmethoden wenigstens mit einigen Strichen zu skizzieren. ${ }^{1}$

Von Eugen Varga läßt sich sagen, daß er das Leben eines kommunistischen Wissenschaftlers vorgelebt hat. Für ihn bildeten konsequente Parteinahme für die Sache der Arbeiterklasse und gründliche wissenschaftliche Analyse immer eine Einheit. Für ihn war die politische Ökonomie wie für die Klassiker des Marxismus-Leninismus immer eine Wissenschaft, die sich als Anleitung zum Handeln für den revolutionären Klassenkampf des Proletariats verstehen muß.

Dabei würde man der Arbeit Eugen Vargas nicht gerecht, wollte man nur seine Beiträge auf dem Gebiete der Theorie der allgemeinen Krise, der Krisentheorie und der aktuellen Wirtschaftseinschätzung hervorheben. Sein Werk umfaßt grundlegende Aufsätze zu methodologischen Fragen in den Arbeiten der Klassiker, zur Theorie der Lage der Arbeiterklasse, zur Ungleichmäßigkeit der Entwicklung der imperialistischen Länder und zu vielen anderen Fragen der politischen Ökonomie des Kapitalismus. Aber auch auf dem Gebiet der politischen Ökonomie des Sozialismus - vor allem zu Agrarproblemen - hat Eugen Varga gerade in seiner Schaffensperiode zwischen den beiden Weltkriegen Wichtiges geleistet.

Eugen Varga war ein hervorragender Kenner der Werke der Klassiker des MarxismusLeninismus. Was ihn aber vor allem auszeichnete, war das ständige erfolgreiche Bemühen, die Lehren der Klassiker auf die Analyse der konkreten Situation anzuwenden. Er entsprach damit der Forderung Lenins nach einer ,,konkreten Analyse in konkreten Situationen" 2 . Eugen Varga hielt nichts von einer bloßen Wiederholung der Thesen der Klassiker ohne eine gründliche Analyse der jeweiligen Situation. In einer Auseinandersetzung mit seinen Kritikern formulierte er im Jahre 1925: „Ich sehe eben die Aufgabe eines wirklichen Marxisten nicht darin, die Marxschen Grundlehren immer von neuem fehlerlos herzusagen, sondern diese Lehren, auf die - seit deren Niederschrift so stark modifizierte - gegenwärtige Wirklichkeit selbständig sinngemäß anzuwenden." 3 Varga bemühte sich immer, ein möglichst umfassendes Bild von den tatsächlichen Vorgängen in der Wirtschaft und Politik des Kapitalismus zu erhalten und diese in ihrer Wechselwirkung zu analysieren. Die Einheit von empirischer und theoretischer Analyse ist ein besonderer Wesenszug der wissenschaftlichen Arbeitsmethode Eugen Vargas. Besonders hervorzuheben ist in diesem Zusammenhang noch ein weiteres Moment. Eugen Varga gab ein Beispiel dafür, wie die bürgerliche Statistik möglichst umfassend für eine marxistische Analyse zu nutzen ist. Dabei standen ihm zu seiner Zeit viel weniger statistische Grunddaten zur Verfügung als heute.

Gestützt auf eine gründliche Analyse, suchte Eugen Varga immer an bestimmten

1 Jürgen Kuczynski, der sich um die Propagierung der Werke Eugen Vargas in unserer Republik besonders verdient gemacht hat, legte eine interessante Studie über „Die Schule Eugen Vargas“ vor. Sie ist abgedruckt in: J. Kuczynski, Studien zu einer Geschichte der Gesellschaftswissenschaften, Bd. 7, Berlin 1977.

2 W. I. Lenin, Kommunismus, in: Werke, Bd. 31, Berlin 1959, S. 154.

3 E. Varga, Wirtschaft und Wirtschaftspolitik im IV. Vierteljahr 1924, in: Internationale Pressekorrespondenz, Deutsche Ausgabe, 5. Jg., Wien 1925, Nr. 21, S. 278.

Dieser wie auch alle anderen zitierten "Vierteljahresberichte" sind in der 5bändigen Faksimileausgabe (Hrsg. Jörg Goldberg), Berlin (West) 1977, enthalten. Vgl. hierzu Fußnote 60! In den folgenden Fußnoten wird die verkürzte Quellenangabe E. Varga, „Wirtschaft und Wirtschaftspolitik ...“, a. a. O. verwendet. 
Schnittpunkten der Entwicklung des Kapitalismus eine theoretische Verallgemeinerung. Er gab sich nicht damit zufrieden, nur kurzfristige Einschätzungen zu liefern, obwohl diese bereits einen eigenen hohen Aussagewert besaßen. Hervorstechende Ergebnisse erzielte er dabei auf den Gebieten der Theorie der allgemeinen Krise und der Krisentheorie. Berühmt geworden sind seine Einschätzungen des historischen Niederganges des Kapitalismus seit dem ersten Weltkrieg, der zeitweiligen relativen Stabilisierung des Kapitalismus in den 20er Jahren, des zyklischen Verlaufs nach der "Großen Krise" (mit seiner Einschätzung der Depression besonderer Art) und der weiteren Entwicklung des Imperialismus am Vorabend des zweiten Weltkrieges. Hier erbrachte Eugen Varga den Beweis, daß eine gründliche marxistisch-leninistische Analyse zu wertvollen prognostischen Einschätzungen gelangen kann, zu denen die bürgerliche Wissenschaft infolge ihrer klassenmäßigen Beschränktheit nicht fähig ist. Seine Voraussagen wurden in der kommunistischen Bewegung hoch eingeschätzt und haben sogar dem Klassenfeind widerwillige Anerkennung abgerungen.

Wenn man von dieser erfolgreichen Forschungsarbeit Eugen Vargas spricht, muß man sogleich noch etwas hinzufügen. Eugen Varga zeichnete sich auch dadurch aus, da $\beta$ er seine Forschungsergebnisse nach einer gründlichen Analyse mit Entschiedenheit und wissenschaftlicher Standhaftigkeit vertrat, auch wenn die Wahrheit manchmal dem Wunschdenken und falschverstandener revolutionärer Ungeduld widersprach. Berühmt geworden sind die Äußerungen Vargas an die Adresse der Kritiker seiner Einschätzung der relativen Stabilisierung des Kapitalismus. Eugen Varga sagte damals: „Wir haben in unseren Berichten and weltwirtschaftlichen Arbeiten wiederholt auf die immanenten und bewußten Tendenzen zu einer Neustabilisierung des Kapitalismus hingewiesen. Manchmal wurde uns dies von Genossen, die von der Möglichkeit einer gewissen Stabilisierung nicht einmal hören wollten, von Genossen, die in ihrer revolutionären Ungeduld den Zusammenbruch des Kapitalismus in unmittelbarer Nähe sahen, sehr übel genommen. So insbesondere auf dem III. und V. Kongreß der Kommunistischen Internationale. Ich wurde ,Rechter', ein ,Opportunist' genannt. Mit Unrecht! Es gibt keine, linke‘ oder ,rechte Analyse; es gibt keine ,opportunistische' oder ,revolutionäre' Perspektive. Es gibt nur ,richtige‘ oder ,unrichtige' Analysen; eine richtige oder eine unrichtige Perspektive. Und mag sich jemand für einen noch so guten Revolutionär halten, weil er die Perspektive des Sieges des Proletariats ständig in kürzester Zeit vor sich sieht: Eine erfolgreiche revolutionäre Politik läßt sich nur auf Grundlage einer richtigen, den Tatsachen entsprechenden Analyse und einer darauf sich gründenden Perspektive erreichen." 4

Natürlich sind in den Arbeiten Eugen Vargas auch Fehler enthalten, und in manchen Prognoseeinschätzungen der Entwicklung des Kapitalismus hat er geirrt. Aber hinsichtlich der Prognosefehler Eugen Vargas ist Jürgen Kuczynski völlig zuzustimmen, wenn er sagt: „Natürlich hat Varga auch Prognosefehler gemacht - sind wir doch immer noch erst am Anfang der Prognosewissenschaft, was die nichtsozialistische Wirtschaft betrifft ... Aber wenn wir überhaupt einen sinnvollen Anfang gemacht haben, dann verdanken wir das der unermüdlichen Pionierarbeit Vargas - echter, Mut erfordernder Pionierarbeit, denn jede solcher Prognosen unterliegt zwei Gefahren: der Widerlegung durch die Wirklichkeit und dem Angriff derjenigen, denen die Prognose ,nicht gefällt'“..5

4 E. Varga, Vierteljahresbericht $I / 1925$, in: Wirtschaft und Wirtschaftspolitik ..., a. a. O., Bd. 2.

5 Jürgen Kuczynski, Die Schule Eugen Vargas, in: Studien zu einer Geschichte der Gesellschaftswissenschaften, Bd. 7, a. a. O., S. 35. 
Eugen Varga hat auch eigene Fehler eingestanden und unhaltbare Thesen korrigiert. Aber manche der theoretischen Auffassungen Eugen Vargas, die seine Kritiker als falsch bezeichneten, haben mittlerweile ihre Bestätigung gefunden bzw. sind heute als wichtige Beiträge zum weiteren wissenschaftlichen Meinungsstreit einzuschätzen. Eugen Varga setzte sich dafür ein, sich den neuen Prozessen in der Entwicklung des Kapitalismus zuzuwenden, neue Fragen aufzuwerfen und undogmatisch an deren Analyse heranzugehen. Nichts war ihm mehr verhaßt als leeres Wortgeklingel und dogmatische Engstirnigkeit. Noch in der letzten Arbeit, die im Jahre seines Todes veröffentlicht wurde, in den „Beiträgen zu Problemen der politischen Ökonomie des Kapitalismus", sagt er über die Dogmatiker: „... die nicht dem Schema entsprechenden neuen Fakten werden ignoriert, statt sie zu untersuchen und die für den gegenwärtigen Kapitalismus typischen neuen Erscheinungen zu analysieren. Die Dogmatiker versuchen, mit einzelnen untypischen Fakten zu beweisen, $\mathrm{da} ß$ alle Erkenntnisse von Marx unverändert gültig sind. Wie W. I. Lenin sagte, lassen sich bei der Kompliziertheit der kapitalistischen Gesellschaft immer einzelne Fakten ausfindig machen, um eine beliebige Theorie zu beweisen. “6

Und noch etwas zeichnet die Arbeiten Eugen Vargas aus: ihre allgemeine Verständlichkeit und damit ihre Popularität innerhalb der Arbeiterbewegung. Eugen Varga verstand seine Arbeiten immer als eine Anleitung zum Handeln für die Arbeiterklasse und wirkte selbst als hervorragender Propagandist des Marxismus-Leninismus.

In diesem Geist eines marxistischen Wissenschaftlers und mit diesen Arbeitsmethoden hat Eugen Varga über den Kreis seiner unmittelbaren Mitarbeiter hinaus eine große Schar von Gesellschaftswissenschaftlern in vielen Ländern maßgeblich beeinflußt. Auch in unserer Deutschen Demokratischen Republik waren und sind seine Schriften wie auch die seiner bedeutenden Mitarbeiter wie E. L. Chmel'nickaja und L. A. Mendel'son von grundlegender Bedeutung für die politökonomische Lehre und Forschung.

Es ist leider in dieser Vorbemerkung zu der dreibändigen Ausgabe nicht möglich, die verschiedenen theoretischen Arbeiten Eugen Vargas im einzelnen zu würdigen und auf besondere Ergebnisse der theoretischen Arbeit Eugen Vargas hinzuweisen. Wir wollen hier lediglich auf zwei wichtige Forschungsgebiete Eugen Vargas etwas näher eingehen. Es geht um seinen maßgeblichen Anteil an der Herausarbeitung der Theorie von der allgemeinen Krise des Kapitalismus und um seine umfassende Arbeit auf dem Gebiet der marxistischen Krisentheorie und der Wirtschaftsanalysen.

Man kann ohne Übertreibung Eugen Varga als einen Schöpfer der Theorie von der allgemeinen Krise des Kapitalismus bezeichnen. Den methodologischen Ausgangspunkt bildete jene Fragestellung, mit der Varga seine im Zusammenhang mit dem III. Weltkongre $\beta$ der Komintern verfaßte Schrift „Die Krise der kapitalistischen Weltwirtschaft“ (1921) einleitete: „Unsere Aufgabe ist es nicht, die Krise zu beschreiben, sondern zu untersuchen, inwiefern diese Krise sich von den gewöhnlichen Krisen unterscheidet: zu untersuchen, ob die gegenwärtige Krise eine ebenso vorübergehende Erscheinung ist wie alle bisherigen Krisen, wie die meisten Kapitalisten und Sozialdemokraten behaupten, oder aber, ob sie

6 E. Varga, Beiträge zu Problemen der politischen Ökonomie des Kapitalismus, in: Bd. 3 dieser Ausgabe, S. 103. 
einen Schritt zur vollständigen Zersetzung der kapitalistischen Gesellschaftsordnung bedeutet ..." "7

„Die gegenwärtige Wirtschaftskrise“, so schrieb Varga in der gleichen Arbeit, ,ist keine gewöhnliche Überproduktionskrise, wie sie der Kapitalismus seit seinem Bestehen wohl ein Dutzend überdauert hat. Sie ist eine Folge jener Veränderungen, welche in der Weltwirtschaft während des Weltkrieges vor sich gegangen sind.“8 „Das weitere Schicksal des Kapitalismus (ist) aufs engste mit der Stärke des revolutionären Kampfes des Proletariats verknüpft. Bei der Beurteilung der Grundfrage: Überwindet der Kapitalismus die durch den Weltkrieg verursachte schwere Erschütterung oder nicht, mußte auch dieser Faktor in Betracht gezogen werden." 9

Diese Fragestellungen: Was ist an der jeweiligen Erscheinung zeitweilig, was sind langfristige Veränderungen im Gesamtzustand des Weltkapitalismus, in den Grundtendenzen seiner Entwicklung? greift Varga in vielen seiner Schriften immer wieder auf, und das Suchen nach möglichst exakten Antworten bringt immer wieder neue und reifere theoretische Gedanken hervor. Es ist eine beispielhafte Anwendung des marxistischen Prinzips der Einheit und Wechselwirkung von konkreter Analyse und theoretischer Verallgemeinerung. Varga wies dabei auf die außerordentliche Schwierigkeit einer solchen Aufgabe hin, aus einer konkreten Situation heraus ,ein allgemeines Urteil“ über die Entwicklung des Kapitalismus zu fällen, ,da die Erscheinungen sehr kompliziert sind und einander entgegengesetzte Strömungen sich im Gesamtbild kreuzen “, die es stets gelte ,gegeneinander abzuwägen". 10

Diese Schwierigkeiten, die unvermeidlich Unvollkommenheiten und Einseitigkeiten der Einschätzungen und auch Fehlurteile hervorbringen, waren Varga durchaus bewußt. Er selbst war deshalb immer wieder um die Überprüfung seiner eigenen Analysen, um die Vervollkommnung und Korrektur seiner Schlußfolgerungen bemüht.

So fällt auf, daß besonders in den Arbeiten der 20er Jahre die kurzfristigen Erscheinungen der konkreten Situation noch recht unscharf von langfristigen Faktoren und Grundprozessen abgehoben sind, daß die Idee einer dauernden, nicht mehr überwindbaren Krise eine ganze Zeitlang faktisch mit der Wirtschaftskrise und ihren zyklischen Momenten identifiziert oder unmittelbar damit verflochten ist u. a. m. Erst später - stets aus der Analyse neuer Erscheinungen in der Wirklichkeit abgeleitet -- schälen sich bei Varga die spezifischen Begriffe und Kategorien der allgemeinen Krise des Kapitalismus, ihre Triebkräfte und Merkmale deutlicher heraus.

Logisch ist daher auch, daß das Problem der Unterschiede und qualitativer Veränderungen im Verlauf der allgemeinen Krise des Kapitalismus - vor allem also die Frage der Etappen und der Unterschiede zwischen ihnen erst nach dem zweiten Weltkrieg für Varga ins Blickfeld rückt.

Eine erste grundlegendere Ausarbeitung erfuhr die Problematik der allgemeinen Krise des Kapitalismus in Vargas Schrift „Die Niedergangsperiode des Kapitalismus“ (1922). Dieser erste theoretische Entwurf für das Erfassen der neuen Qualität der Existenzbedingungen des Kapitalismus nach dem ersten Weltkrieg und der Oktoberrevolution stellt eine große wissenschaftliche Leistung dar, die sich in ihrer Bedeutung für die Strategie und

7 E. Varga, Die Krise der kapitalistischen Weltwirtschaft, in: Bd. I dieser Ausgabe, S. 180.

8 Ebenda, S. 184.

9 Ebenda, Vorwort zur zweiten Aufl., S. 181.

10 Ebenda, S. 278. 
Taktik des Klassenkampfes in der Folgezeit, bei den Diskussionen um den Charakter der Stabilisierungsperiode und um die Besonderheiten der Wirtschaftskrise 1929 bis 1933 voll bewährte. Varga war durch die Analyse der Tatsachen darauf gestoßen, da $B$ sich in den Jahren 1921/1922 und dann stärker in den folgenden Jahren Anzeichen einer Verbesserung der wirtschaftlichen Lage in den kapitalistischen Ländern zeigten. Was bedeutet dies? Handelte es sich um eine Neustabilisierung des Kapitalismus? Welchen Charakter hat diese Stabilisierung? Führt sie zu einer Wiederherstellung seines alten Gleichgewichts oder geht es um eine zeitweilige Erscheinung? Ist der Kapitalismus überhaupt zu einer wenn auch zeitweiligen Stabilisierung seiner Lage fähig? Um diese Fragen entwickelten sich scharfe Diskussionen auch innerhalb der Komintern, wobei sowohl rechtsopportunistische wie auch linksopportunistische Kritiker von Vargas Auffassungen auftraten.

Varga schlußfolgerte vor allem aus seinen Analysen Mitte der 20er Jahre die Neustabilisierung des Kapitalismus. Das ökonomische Kriterium war für ihn - neben anderen Faktoren - der Übergang zur erneuten erweiterten Reproduktion. Politisch war für Varga die Tatsache maßgebend, daß vor allem in Mitteleuropa keine akut revolutionäre Situation mehr vorhanden war.

Gestützt auf seine Erkenntnisse über den Eintritt des Kapitalismus in eine qualitativ neue Periode seiner historischen Entwicklung, eben der Niedergangsperiode des Kapitalismus, die Periode seiner allgemeinen Krise, unterstreicht Varga zugleich unzweideutig den zeitweiligen, relativen Charakter dieser Neustabilisierung und leistet damit einen hervorragenden Beitrag sowohl zur Einschätzung einer komplizierten historischen Situation als auch zum weiteren Ausreifen der Elemente einer Theorie der allgemeinen Krise des Kapitalismus.

Varga benutzt längere Zeit zur generellen Kennzeichnung der Situation des Kapitalismus noch die verschiedensten Begriffe. Er spricht vom „Verfallstadium des Kapitalismus“, von der „Periode der ständigen Krise“, von der „,ununterbrochenen Krise“, der „Periode der langandauernden Krise im Weltmaßstabe“ oder auch nur von der „Krisenperiode des Kapitalismus". Seiner oben genannten Schrift gibt er, wie bereits erwähnt, den Titel „Die Niedergangsperiode des Kapitalismus“.

In Verbindung mit der Suche nach dem treffendsten Begriff wies Varga auf die Kompliziertheit der Entwicklungsprozesse hin und warnte vor vereinfachten Vorstellungen: „Diese Züge sind natürlich nicht in allen Ländern der Welt gleich stark vorhanden ... Die Periode der ständigen Krise muß im Weltmaßstab als langandauernd angenommen werden. Innerhalb derselben wird es Zeiten besserer Konjunktur und der akuten Krisen (gemeint sind zyklische Wirtschaftskrisen - d. Hrsg.) geben." ${ }^{11}$ „Der Niedergang des Kapitalismus ist eben kein automatischer Prozeß, sondern eine Kampfperiode mit wechselnden Erfolgen und Mißerfolgen für das kämpfende Proletariat." ${ }^{\text {12 }}$

Bei der Analyse der großen Weltwirtschaftskrise benutzte dann Varga immer häufiger den Begriff der ,allgemeinen Krise des Kapitalismus“, der sich schließlich in den 30er Jahren in den kollektiven Einschätzungen der Komintern, in den Analysen der KPdSU und zahlreicher anderer kommunistischer Parteien endgültig durchsetzte.

In dieser Zeit arbeitet Varga immer präziser eine Bestimmung des Inhalts, des Wesens der allgemeinen Krise des Kapitalismus heraus. Gestützt auf die vielfältigen Erfahrungen der wechselhaften ökonomischen und politischen Entwicklung nach dem ersten Weltkrieg,

11 E. Varga, Die Niedergangsperiode des Kapitalismus, in: Bd. 1 dieser Ausgabe, S. 286.

12 Ebenda, S. 297. 
vor allem auf die Erkenntnisse, die die Weltwirtschaftskrise 1929 bis 1933 vermittelte, formuliert Varga 1933 seine für die damalige Periode wohl reifste Definition: „Die allgemeine Krise ist jene Periode des Imperialismus, in der sich der sterbende Kapitalismus in einen zum Teil bereits gestorbenen Kapitalismus verwandelt hat, in der das kapitalistische Gesellschaftssystem auf einem sechsten Teil der Erde bereits gestürzt ist und der Kampf des Proletariats gegen die Bourgeoisie bereits die Form des Kampfes zweier Wirtschaftssysteme angenommen hat." 13

In den heutigen gesellschaftswissenschaftlichen Diskussionen über die Probleme der allgemeinen Krise des Kapitalismus taucht immer wieder die Frage nach dem Beginn der allgemeinen Krise des Kapitalismus auf, die theoretisch mit der Bestimmung ihres Inhalts eng verknüpft ist. Es geht dabei vor allem um die Frage, ob die allgemeine Krise des Kapitalismus unmittelbar mit der Oktoberrevolution, oder schon vorher, also mit dem Weltkrieg oder gar mit dem Übergang zum Monopolkapitalismus datiert werden kann, in welchem Verhältnis die ,inneren“ Krisenprozesse des Kapitalismus einerseits und der ,äußere“ Einfluß der siegreichen proletarischen Revolution anderseits als Triebkräfte der allgemeinen Krise stehen. Was läßt sich über die Position Vargas dazu sagen?

Erstens sah Varga den Weltkrieg und die Oktoberrevolution in einem sehr engen, in einer konkreten historischen Situation entstandenen, Ursache-Wirkung-Verhältnis. Seine Art der Betrachtung läßt eigentlich keine scholastische Gegenüberstellung zu: „,Der Weltkrieg, den wir als eine besondere äußerst scharfe Krise auffassen können, führte zum Sturz der Bourgeoisie in einem der mächtigsten Staaten der Welt."14

Diese Kennzeichnung des Krieges als eine historische spezifische Form einer das ganze kapitalistische System erfassenden Krise ist besonders heute, für die Analyse der dritten Etappe der allgemeinen Krise wichtig, deren Besonderheit bekanntlich darin besteht, da $\mathrm{B}$ sie sich ohne einen Weltkrieg herausbildete. Varga beschäftigt sich mit dieser Frage, wenn auch nicht mehr sehr umfassend, in einigen seiner Arbeiten nach dem zweiten Weltkrieg, besonders Anfang der 60er Jahre.

Der zweite Aspekt der Vargaschen. Betrachtungsweise ist die Unterstreichung des Zusammenhangs von allgemeiner Krise und Monopolkapitalismus bzw. Imperialismus. „Der Imperialismus, der monopolistische Kapitalismus ist der historisch konkrete Weg, der auf Grundlage der allgemeinen Bewegungsgesetze der kapitalistischen Gesellschaftsordnung zur allgemeinen Krise des Systems, zu einem revolutionären Zusammenbruch führen muß."15

Wiederholt berief sich Varga bei der Ausarbeitung von Fragen der allgemeinen Krise auf Lenins Imperialismustheorie: „Wichtig ist für uns in diesem Zusammenhang nur die Feststellung Lenins, daß der Imperialismus sterbender Kapitalismus ist. Dies bedeutet, daß die Periode der allgemeinen Krise des Kapitalismus durch keine festen Grenzen, durch keine chinesische Mauer vom Imperialismus geschieden ist. “16 „Den Monopolkapitalismus von der allgemeinen Krise zu trennen oder gegenüberzustellen, ist undialektisch und unrichtig.“"17 In diesem Sinne ist wohl auch Vargas polemische Bemerkung in der Diskussion im Jahre 1947 zu verstehen, daß für ihn die erste Etappe der allgemeinen Krise ,mit

13 E. Varga, Vierteljahresbericht III/1933, in: Wirtschaft und Wirtschaftspolitik ..., a. a. O., Bd. 5.

14 E. Varga, Vierteljahresbericht $\mathrm{I} / 1931$, in: Wirtschaft und Wirtschaftspolitik ..., a. a. O., Bd. 4.

15 E. Varga, Vierteljahresbericht III/1933, in: Wirtschaft und Wirtschaftspolitik ..., a. a. O., Bd. 5.

16 E. Varga, Vierteljahresbericht $\mathrm{I} / 1931$, in: Wirtschaft und Wirtschaftspolitik ..., a. a. O., Bd. 4.

17 E. Varga, Vierteljahresbericht III/1933, in: Wirtschaft und Wirtschaftspolitik ..., a. a. O., Bd. 5. 
der vollen Entwicklung des monopolistischen Stadiums des Kapitalismus zusammenfällt". ${ }^{18}$

Der dritte Aspekt der Vargaschen Betrachtungsweise besteht aber eben darin, die allgemeine Krise nicht nur in ihrem Zusammenhang mit dem monopolkapitalistischen, imperialistischen Stadium des Kapitalismus zu sehen, sondern sie in ihrer qualitativen Spezifik zu erfassen und zu kennzeichnen. Und hier liegt bei Varga der Hauptakzent, der springende Punkt für das Verständnis des Wesens der allgemeinen Krise eindeutig bei der Oktoberrevolution, beim Sturz der Bourgeoisie in einem Teil der Welt, oder allgemeiner ausgedrückt, in der tatsächlichen, realen Verknüpfung einer ,ungewöhnlichen“ Krise mit dem siegreichen revolutionären Kampf des Proletariats und der Schaffung eines neuen, dem Kapitalismus gegenüberstehenden Gesellschaftssystems.

Das ist bereits aus den von Varga formulierten Grundzügen der Niedergangsperiode zu erkennen. Später, im Jahre 1927, hebt Varga in seiner Schrift „Die ersten zehn Jahre der Niedergangsperiode des Kapitalismus" die entscheidende Qualität der neuen Existenzbedingungen des Kapitalismus mit den Worten hervor: „Der heutige Kapitalismus ist nicht mehr nur ein ,sterbender Kapitalismus', sondern ein zum Teil gestorbener Kapitalismus. "19 In diesem Sinne spricht er auch von der allgemeinen Krise des Kapitalismus als einer „besonderen Periode des Imperialismus“. ${ }^{20}$

Sowohl die innere tiefe Krise des kapitalistischen Systems als auch die tatsächliche revolutionäre Überwindung des Kapitalismus in einem oder in mehreren Ländern waren - als dialektische Einheit betrachtet - somit für Varga die wichtigsten qualitativen Bestimmungsfaktoren der allgemeinen Krise des Kapitalismus und die Haupttriebkräfte ihrer Entwicklung.

Diese Wesensbestimmung der allgemeinen Krise des Kapitalismus schält sich in Vargas Arbeiten immer deutlicher heraus. Sie wird zugleich das methodologische Grundprinzip seiner weiteren Forschungen, die schließlich - nach dem zweiten Weltkrieg - ihre prägnanteste Zusammenfassung in seiner Schrift „Der Kapitalismus des zwanzigsten Jahrhunderts" finden, wo auch die Charakterisierung der Etappen der allgemeinen Krise, die Bestimmung der Haupttriebkräfte für die Herausbildung einer jeweils neuen Etappe von diesen dargestellten theoretischen Grundpositionen aus vorgenommen wird. Varga periodisiert:

- die Periode vor dem ersten Weltkrieg;

- die erste Etappe der allgemeinen Krise;

- die zweite Etappe der allgemeinen Krise;

- die neue (dritte) Etappe der allgemeinen Krise.

Vargas Stärke war zweifellos die organische Verknüpfung von Ökonomie und Politik in buchstäblich allen seinen Arbeiten, und sicher führte ihn gerade diese Fähigkeit zum Erkennen der allgemeinen Systemkrise des Kapitalismus, deren Ursachen und Wesen eben nur in dieser Einheit zu begreifen sind. Varga knüpfte an seine ökonomischen Untersuchungen stets die Frage, welche Probleme und Möglichkeiten sich daraus für den

18 Diskussion über das Buch „Veränderungen in der kapitalistischen Wirtschaft im Gefolge des zweiten Weltkrieges“ von Eugen Varga, 1. Beiheft zur ,Sowjetwissenschaft", Berlin (1948), S. 127.

19 E. Varga, Die ersten zehn Jahre der Niedergangsperiode des Kapitalismus, in: Die Kommunistische Internationale, Hamburg-Berlin, 8/1927, S. 2206.

20 E. Varga, Vierteljahresbericht IV/1933, in: Wirtschaft und Wirtschaftspolitik ..., a. a. O., Bd. 5. 
revolutionären Kampf der Arbeiterklasse ergeben und lenkte seine politischen Schlußfolgerungen vor allem in diese Richtung. Von seinen „Vierteljahresberichten“ sagte er, daß es ihr Zweck ist, ,den Genossen eine skizzenhafte Übersicht der Zusammenhänge zwischen den wirtschaftlichen, wirtschaftspolitischen und außenpolitischen Ereignissen in den wichtigeren kapitalistischen Ländern zu vermitteln. "21 Zugleich unterstrich Varga immer wieder die Notwendigkeit, gerade im Interesse einer erfolgreichen revolutionären Politik „die Weltlage theoretisch unvoreingenommen ohne Illusionen zu untersuchen". 22

Es versteht sich, daß Varga als Politökonom seine Hauptaufmerksamkeit auf die Ökonomie konzentrierte. Dabei ist bemerkenswert, daß solche Kennzeichnungen stets aus der Analyse jeweils konkreter Situationen, in denen sich die Widersprüche verschärften und miteinander verknüpften, abgeleitet werden.

Diese bei Varga stark ausgeprägte engste Verknüpfung von konkreter Analyse und allgemein-theoretischen Schlußfolgerungen, auf die er in der Regel später prüfend und teils bestätigend, teils korrigierend zurückkam, muß man berücksichtigen, wenn man sich mit seinen theoretischen Grundgedanken heute beschäftigt, nach den Erfahrungen von mehr als drei Jahrzehnten kapitalistischer Entwicklung nach dem zweiten Weltkrieg mit wesentlichen Veränderungen der Existenzbedingungen des kapitalistischen Systems, aus denen viele neue Erscheinungen resultieren, die Varga nur noch teilweise verfolgen konnte.

Versuchte man, die wichtigsten der in Vargas Arbeiten als ökonomische Merkmale der allgemeinen Krise des Kapitalismus hervorgehobenen Erscheinungen und Tendenzen einmal ohne Anspruch auf Systematik aufzuzählen, so sind das:

1. der chronische Überfluß an fixem Kapital;

2. das Entstehen einer chronischen (strukturellen, technologischen) Arbeitslosigkeit;

3. das Fehlen des weltwirtschaftlichen Gleichgewichts bzw. das Entstehen einer chronischen weltwirtschaftlichen Disproportionalität (vor allem als Störung der Arbeitsteilung zwischen imperialistischen ,Mutter"ländern und Kolonien, als Asynchronität der zyklischen Entwicklung, als Ungleichmäßigkeit der Entwicklung der kapitalistischen Länder und starke Verschiebung der Kräfteverhältnisse zwischen ihnen);

4. die chronische Agrarkrise;

5. die Einengung des inneren und äußeren Marktes und die tendenziell chronische Verschärfung des Marktproblems;

6. die Tendenz des Rückgangs der internationalen Arbeitsteilung;

7. die Inflation, der Zusammenbruch des Goldstandards und die Labilität der Devisenkurse;

8. die Tendenz, daß alle ökonomischen Widersprüche, die in den zyklischen Krisen eine zeitweilige Lösung erfahren, eine mehr oder weniger ständige Schärfe erhalten und der Krisenzyklus selbst deformiert wird (vor allem, indem die Aufschwungsphasen tendenziell kürzer, die Belebungs- und Depressionsphasen länger und qualvoller werden).

Für das Verständnis der Vargaschen Konzeption verdient besondere Hervorhebung, was er selbst als „ökonomische Wurzel der allgemeinen Krise des Kapitalismus“ bezeichnete: „Am schärfsten tritt in der Periode der allgemeinen Krise der Widerspruch zwischen dem durch die Konkurrenz erzwungenen Bestreben des Kapitals - ungeachtet der monopolistischen Verfaulung - nach einer unbegrenzten Ausdehnung der Produktion und der

21 E. Varga, Vierteljahresbericht II/1929, in: Wirtschaft und Wirtschaftspolitik ..., a. a. O., Bd. 4.

22 E. Varga, Die Niedergangsperiode des Kapitalismus, in: Bd. 1 dieser Ausgabe, S. 283. 
durch die inneren Bewegungsgesetze der kapitalistischen Produktionsweise relativ immer enger beschränkten Konsumtionskraft der kapitalistischen Gesellschaft hervor. Während dieser Widerspruch in den früheren Perioden des Kapitalismus nur in den periodisch wiederkehrenden Wirtschaftskrisen zum offenen Ausbruch kam, zeigt er in der Periode der allgemeinen Krise des Kapitalismus die Tendenz, chronisch scharf zu werden. Die Aufnahmefähigkeit des kapitalistischen Absatzmarktes genügt selbst in den Hochkonjunkturphasen nicht, um eine volle Ausnutzung des Produktionsapparates zu ermöglichen . . . Die andere Seite der gleichen Erscheinung: Die Aufnahmefähigkeit des kapitalistischen Absatzmarktes genügt nicht, um dem ganzen Proletariat Arbeitsgelegenheit zu verschaffen." 23

Und Varga faßt diesen seinen Gedanken in der von ihm später in verschiedenen Varianten wiederholten Formulierung zusammen: „Unausgenützte Produktionsmittel und unausgenützte Arbeitskräfte, Überfluß an Kapital bei Überfluß an Bevölkerung - wie Marx sagt - ist unseres Erachtens das wichtigste ökonomische Merkmal der allgemeinen Krise des Kapitalismus. “24

Betrachtet man diese von Varga dargestellten Zusammenhänge vom heutigen Standpunkt, so kann man feststellen, daß sie sich vielfach bestätigt und ihre Gültigkeit bis in die Gegenwart behalten haben. Auch der heutige, der Kapitalismus der 70er Jahre ist gezeichnet von diesen ökonomischen Grundkonflikten und Grundprozessen der allgemeinen Krise. Allerdings darf man bei dieser Feststellung keinesfalls stehenbleiben, da die konkreten Zusammenhänge, die Ursachen und Wirkungen, die quantitativen Ausmaße, der Stellenwert der einzelnen ökonomischen Erscheinungen und Tendenzen usw. sich heute doch beträchtlich von den 20er und 30er Jahren unterscheiden und überhaupt in den einzelnen Entwicklungsetappen der allgemeinen Krise Veränderungen erfahren haben. Und gerade dieses Erfassen der konkreten Situation, der Veränderungen, der neuen Momente ist zumindest ebenso wichtig wie das Hervorheben der Parallelen zu früheren Perioden. Nur so können auch richtige Schlußfolgerungen für die jeweiligen Bedingungen des Klassenkampfes gezogen werden.

Varga stieß selbst auf diese Problematik im Ergebnis seiner Untersuchungen über die Veränderungen des Kapitalismus in und nach dem zweiten Weltkrieg und über die neuen ökonomischen Prozesse, die sich nach der Wiederherstellungsperiode in der ökonomischen Entwicklung der kapitalistischen Länder in den 50er Jahren zeigten.

In seiner Schrift „,Der Kapitalismus des zwanzigsten Jahrhunderts“ sind für Varga die Wirkungen der großen Veränderungen des internationalen Kräfteverhältnisses auf die inneren Prozesse und Gesetzmäßigkeiten des Kapitalismus der Ausgangspunkt und der entscheidende Aspekt seiner Betrachtung. Faktisch stößt Varga hier auf das Problem der Anpassung des Kapitalismus, ohne es allerdings schon als selbständige Frage und in seinen Hauptrichtungen auszuarbeiten. Varga behandelt z. B. die neuen Momente der Frage Krieg und Frieden: , Die dem Imperialismus eigenen Gesetzmäßigkeiten drängen nach wie vor zum Krieg. Seit Beendigung des zweiten Weltkrieges fanden bereits mehr als zehn „lokale“ Kriege statt (Korea, Vietnam, Suez, Algerien usw.). Aber die Entfesselung eines neuen Weltkrieges ist für die Imperialisten gegenwärtig nicht so einfach. In unserèr Zeit gibt es Kräfte, die einen neuen Weltkrieg zu verhindern vermögen. “25

23 E. Varga, Vierteljahresbericht I/1931, in: Wirtschaft und Wirtschaftspolitik ..., a. a. O., Bd. 4.

24 Ebenda.

25 E. Varga, Der Kapitalismus des zwanzigsten Jahrhunderts, in: Bd. 3 dieser Ausgabe, S. 61 . 
In einem ähnlichen Sinne geht Varga auf den prinzipiellen Unterschied in den Beziehungen zwischen schwachentwickelten und imperialistischen Ländern heute und in der Vorkriegsperiode ein. ${ }^{26}$ Besondere Aufmerksamkeit widmet er dem staatsmonopolistischen Kapitalismus, der sich, wie er schreibt, nunmehr vollständig entwickelt hat und zur Entwicklungsform des Kapitalismus unter den Bedingungen seiner allgemeinen Krise geworden ist. ${ }^{27}$

Varga beschäftigt sich mit der schnellen Einführung der neuen Technik, die durch den hohen und sich weiterentwickelnden Grad der Monopolisierung und die Einbeziehung des Staates in die ökonomischen Prozesse ermöglicht wird und auf diese wiederum stimulierend zurückwirkt. Varga macht darauf aufmerksam, daß die heutige Technik viele neue Züge trägt, daß sich neue Energiequellen, die Elektroenergie und die Kernkraft und die darauf basierenden Antriebskräfte durchsetzen, daß ganze Maschinensysteme zum Einsatz kommen und vollautomatische Fabriken zunehmend errichtet werden. ${ }^{28}$ Varga knüpft hier an seine Grundposition zur Entwicklung des wissenschaftlich-technischen Fortschritts an, wie er sie in den 30er Jahren formulierte: „Alle ... Daten zeigen klar, daß von einer Stagnation oder gar von einer Verkrüppelung der Produktivkräfte der kapitalistischen Weltwirtschaft in der Periode der allgemeinen Krise keine Rede sein kann. Wenn auch in verlangsamten Tempo, wenn auch nach Ländern und Produktionszweigen höchst ungleichmäßig, wenn auch stark durch die Kriegsvorbereitungen deformiert, so geht doch die Entwicklung der Produktivkräfte weiter. Die allgemeine Krise des Kapitalismus macht sich nicht in einer Stagnation der Entwicklung der Produktivkräfte geltend, sondern in der Unfähigkeit des Kapitalismus, die vorhandenen und unter dem Druck der Konkurrenz stetig vergrößerten Produktivkräfte auszunutzen. “29

In Verbindung mit seinen Beobachtungen über die Entwicklung der Produktivkräfte nach dem Weltkrieg präzisiert Varga seine Auffassungen zu verschiedenen politökonomischen Problemen und setzt sich dabei mit dogmatischen Entwicklungen marxistischer Standpunkte auseinander. Hier ist seine Schrift „Beiträge zu Problemen der politischen Ökonomie des Kapitalismus" von außerordentlichem Interesse. Sicher ist es nicht zufällig, daß sich Varga dabei u. a. mit dem Problem des Umfangs bzw. der Einengung des kapitalistischen Marktes beschäftigt. Er hebt hervor, daß man auf keinen Fall davon ausgehen kann, daß etwa der Umfang des Marktes oder auch der Produktion im Kapitalismus sich in der Nachkriegsperiode verringert habe, wie das verschiedentlich (z. B. in Arbeiten J. W. Stalins) vorausgesagt wurde. „Die Grenzen des Marktes ergeben sich aus den Produktionskapazitäten und den Möglichkeiten für den Absatz der Waren. Beide Seiten dieses Verhältnisses können sich entgegengesetzt entwickeln. Das Marktvolumen kann absolut zunehmen und gleichzeitig im Verhältnis zu den wachsenden Produktionskapazitäten relativ schrumpfen, das heißt, die Absatzschwierigkeiten können trotz zunehmenden Marktvolumens größer werden. Das ist nicht nur möglich, sondern in der Geschichte des Kapitalismus die bestimmende Tendenz. “30

26 Vgl. ebenda, S. 63.

27 Vgl. ebenda, S. 70.

28 Ebenda, S. 68-69.

29 E. Varga, 20 Jahre Kapitalismus und Sozialismus, Straßburg-Moskau 1938, S. 35.

30 E. Varga, Beiträge zu Problemen der politischen Ökonomie des Kapitalismus, in: Bd. 3 dieser Ausgabe, S. 222. 
In ähnlicher Weise untersuchte Varga die Entwicklung des Reallohnes und anderer Faktoren der Lage der Arbeiterklasse und polemisierte gegen den Dogmatismus in der Darstellung des Gesetzes der absoluten Verelendung der Arbeiterklasse, wie er sich vor allem in der Negierung von Tatsachen und der Unterschätzung der Möglichkeiten eines erfolgreichen Kampfes der Arbeiterklasse gegen die Wirkungen dieses Gesetzes äußerte. ${ }^{31}$

Besonders faszinierend bei der Lektüre der Werke Vargas sind eindeutig seine Prognosen. In unseren Tagen, da sich eine spezielle Prognosewissenschaft zu entwickeln beginnt, können die Werke Vargas als methodologische Lehrbücher, als großartige Entwürfe und Muster marxistischer Prognosen genutzt werden. Sie machen vor allem deutlich, daß die gründliche Beherrschung der marxistischen Theorie, das allseitige Studium der Tatsachen und die tiefe Einsicht in die Probleme des lebendigen Klassenkampfes, in die Rolle des subjektiven Faktors die entscheidenden Grundlagen einer marxistischen Gesellschaftsprognose darstellen.

Viele der Vargaschen Vierteljahresberichte enthalten einen speziellen Abschnitt mit der Überschrift „Die Perspektive der weiteren Entwicklung“. Hier, bzw. unmittelbar eingefügt in die Analyse der wirtschaftlichen und meist auch politischen Situation, formuliert Varga Voraussagen, die er aus dem empirischen Material und aus theoretischen Schlußfolgerungen ableitet. Sie tragen in den Vierteljahresberichten meist kurz- oder mittelfristigen Charakter, prognostizieren die nächste Phase des Wirtschaftszyklus, das Herannahen qualitativer Veränderungen der Situation und beschäftigen sich z. T. auch mit prinzipielleren und längerfristigen ökonomischen und politischen Perspektiven.

So gab Varga, wie bereits erwähnt, eine vorausschauende Einschätzung der auf die Nachkriegskrise folgenden Periode der Stabilisierung. Er sagte ihre Labilität und ihr baldiges Ende durch eine neue Wirtschaftskrise voraus. Berühmt wurden die Vargaschen Voraussagen der großen Weltwirtschaftskrise, die er entgegen und in Polemik mit den bürgerlichen Konjunkturprognosen gab, die nur kurzfristige konjunkturelle Schwierigkeiten zu sehen glaubten.

Nach der wesentlich auf Vargas Studien beruhenden Einschätzung der Komintern im Jahre 1928, daß das Ende der Stabilisierung und eine neue zyklische Krise heranreift, schrieb Varga im Sommer 1929: „Wir können mit Sicherheit behaupten, daß die amerikanische Hochkonjunktur das Jahr 1929 nicht umfassen wird."32 Und Anfang 1930 registrierte Varga den Beginn der Wirtschaftskrise in den Vereinigten Staaten und gab die Prognose: „Es ist daher wahrscheinlich, daß wir nach dem Jahre 1920 in diesem Jahre das erste Mal wieder eine die ganze kapitalistische Welt umfassende Wirtschaftskrise haben werden. “33 Bereits in der Anfangsphase der 1929 ausgebrochenen Krise sagte Varga voraus, daß sich ein kapitalistischer Ausweg aus ihr in jenen deformierten Formen vollziehen wird, die den Zyklus unter den Bedingungen der allgemeinen Krise kennzeichnen, daß eine langwierige Depressionsphase bevorsteht, was sich dann voll bestätigte.

Vargas Prognosen hatten in der von ihm immer allseitiger entwickelten Theorie der allgemeinen Krise des Kapitalismus ihre entscheidende wissenschaftliche Grundlage, z. T. bildeten sie einen organischen Bestandteil dieser Theorie.

31 Ebenda, S. 179-188.

32 Rede auf dem X. Plenum des EKKI im Juli 1929, zit. in: E. Varga, Vierteljahresbericht IV/1929, in: Wirtschaft und Wirtschaftspolitik ..., a. a. O., Bd. 4.

33 Ebenda, S. 287. 
Vor allem gilt das für die aus der Analyse der Weltwirtschaftskrise 1929 bis 1933 und ihrer ökonomischen und politischen Folgen abgeleitete Voraussage über das Bevorstehen eines neuen, des ,zweiten Turnus der Revolutionen“. Aus Vargas Einschätzungen am Beginn und während der Krise geht hervor, daß er zunächst in einer direkteren Verknüpfung mit dieser Krise das Ausreifen revolutionärer Situationen erwartet hatte. Das bestätigte sich nicht, zumindest nicht in der erwarteten Schärfe. Doch der komplizierte Ausgang der Krise, die schwere Depression, die Verschärfung des Kampfes um die Märkte, die bewegten Ereignisse in Ländern wie Deutschland, Frankreich u. a. beschäftigten Varga eben vom Standpunkt dieser Perspektive. Lesen wir Vargas 1934 gegebene hochinteressante Prognose: „Der entscheidende historische Hauptunterschied des heranreifenden zweiten Turnus der Revolutionen gegenüber dem ersten ist, daß die revolutionäre Krise gegenwärtig vor dem zweiten Turnus der Kriege heranreift. Dies bedeutet keinesfalls, daß der zweite Turnus der Revolutionen nicht zeitlich mit dem neuen Weltkrieg zusammenfallen, sich mit ihm verflechten wird. Dies ist sogar höchstwahrscheinlich ... Es wäre voreilig im vorhinein entscheiden zu wollen, ob der zweite Turnus der Revolutionen vor dem Ausbruch des Weltkrieges beginnen wird oder in Verflechtung mit demselben ... Sicher aber ist folgendes: Falls der imperialistische Krieg früher beginnt, als der neue Turnus der Revolutionen, so wird derselbe den Beginn der Revolutionen ungemein beschleunigen . . ."34

Welch hervorragendes Beispiel des Feingefühls Vargas für den widersprüchlichen Ablauf der Geschichte und für seine Fähigkeit, Keime neuer Wechselwirkungen von Krieg und Revolution zu erkennen. In direkter Beziehung dazu steht ein weiteres Prognosebeispiel: Die in den Schlußfolgerungen von Vargas Schrift „Der Kapitalismus des zwanzigsten Jahrhunderts" enthaltene Voraussage der Möglichkeit, einen dritten Weltkrieg zu verhindern: „Ein dritter Weltkrieg zwischen Kapitalismus und Sozialismus wird kaum ausbrechen, wenn die Völker aller Länder aktiv für den Frieden kämpfen und wenn die Frage ,Krieg oder Frieden' auch in den kapitalistischen Ländern von vernünftig denkenden Staatsmännern entschieden wird. "35 Und Varga knüpft daran Gedanken über die Notwendigkeit eines langwierigen Kampfes um diese Frage, über die Schwierigkeiten effektiver Schritte in Richtung einer Abrüstung, über die Wahrscheinlichkeit ,kleiner Kriege“ u. a.

Vargas Prognosen waren keineswegs immer richtig. Er irrte aber weniger hinsichtlich genereller ökonomisch-politischer Entwicklungstendenzen, sondern mehr in bezug auf konkrete Prozesse und Ereignisse, vor allem hinsichtlich ihrer Zeiträume, ihrer Dauer, ihres Ausmaßes usw. So bestätigten sich z. B. Vargas Voraussagen, daß nach dem zweiten Weltkrieg die internationale Arbeitsteilung zurückgehen wird, da $B$ die Arbeitslosigkeit größer sein wird als zwischen den beiden Weltkriegen, nicht. Sicher unterschätzte Varga auch das Ausmaß und die Wirkungen der ökonomisch-technischen Veränderungen in der Landwirtschaft, konnte sich nur in Anfängen mit den neuen Problemen der ökonomischen Entwicklung in der ,dritten Welt" beschäftigen usw.

Das führte zu Fehlprognosen, die Varga oft selbst später mutig korrigierte und die in ihrem Gewicht mit der Größe seiner weitsichtigen wissenschaftlichen Aussagen nicht vergleichbar sind. Die Quelle der Arbeitsweise Vargas, der er stets treu blieb, war seine Überzeugung, da $B$ die lebendige marxistische Theorie keine für alle Zeit gültigen absoluten Wahrheiten kennt und in einem widersprüchlichen Erkenntnisproze $\beta$ zu immer vollkommneren Einsichten in die Gesetzmäßigkeiten der Entwicklung gelangt.

34 E. Varga, Vierteljahresbericht $1 \mathrm{I} / 1934$, in: Wirtschaft und Wirtschaftspolitik ..., a. a. O., Bd. 5.

35 E. Varga, Der Kapitalismus des zwanzigsten Jahrhunderts, in: Bd. 3 dieser Ausgabe, S. 98. 
Ein zweites Gebiet der politischen Ökonomie des Kapitalismus, auf dem Eugen Varga Bedeutendes geschaffen hat, ist die marxistisch-leninistische Krisentheorie. Auch aus heutiger Sicht sind seine wissenschaftlichen Ergebnisse für die Weiterentwicklung der marxistischen Krisentheorie unter den Bedingungen der weiteren Vertiefung der allgemeinen Krise des Kapitalismus von unschätzbarem Wert. Es muß daher eigentlich verwundern, daß es bis heute keine Arbeit gibt, die sich gründlich mit diesem wesentlichen Baustein des Vargaschen Lebenswerkes beschäftigt hat. Als erste wichtige Beiträge zu einer solchen Würdigung können die bereits erwähnte Studie von Jürgen Kuczynski sowie das Vorwort von Jörg Goldberg zu der 5bändigen Faksimileausgabe der Vargaschen Vierteljahresberichte angesehen werden. ${ }^{36}$ Eine umfassende Würdigung und kritische Sichtung der Vargaschen Arbeiten auf dem Gebiet der Krisentheorie steht aber noch aus. Auch in den Werken bekannter marxistischer Krisentheoretiker wie L. A. Mendel'son, I. A. Trachtenberg und Fred Oelßner findet man keine Einschätzung des Vargaschen Beitrages zur marxistisch-leninistischen Krisentheorie. ${ }^{37}$

Dabei gibt gerade der Krisenforscher Eugen Varga ein hervorragendes Beispiel für die umfassende Anwendung und eine Bereicherung der Marxschen Theorie. Eugen Varga war ein hervorragender Kenner der Marxschen Krisentheorie, und er leistete Pionierarbeit bei deren Anwendung auf die Analyse des konkreten wirtschaftlichen Verlaufs in den kapitalistischen Ländern. Von ihm stammt der erste bedeutende Beitrag zur Weiterentwicklung der marxistischen Krisentheorie für die Periode der allgemeinen Krise des Kapitalismus.

Als erstes muß man hervorheben, daß Eugen Varga in den 20er und 30er Jahren bedeutende wissenschaftliche Leistungen bei der Systematisierung und Propagierung der Marxschen Krisentheorie vollbrachte. Bekanntlich war es Karl Marx nicht mehr vergönnt, eine geschlossene Abhandlung über die Krisentheorie zu schreiben. Sein theoretisches Konzept zu diesem Problem hat Marx in den „Grundrissen zur Kritik der politischen Ökonomie“, in den drei Bänden des Werkes „Das Kapital“ und vor allem im zweiten Band der „Theorien über den Mehrwert" niedergelegt. Weitere grundlegende Beiträge zur marxistischen Krisentheorie finden sich in den Arbeiten von Friedrich Engels, so vor allem in seiner Schrift „Herrn Eugen Dührings Umwälzung der Wissenschaft” (AntiDühring) sowie in mehreren Schriften W. I. Lenins. Es blieb aber eine wesentliche wissenschaftliche Aufgabe, diese Grundthesen der Klassiker des Marxismus-Leninismus in einem geschlossenen theoretischen Gebäude zusammenzutragen und zu systematisieren, um sie für die Forschung und Lehre und den Kampf der internationalen Arbeiterklasse allseitig nutzbar zu machen. Was mitunter in Vergessenheit gerät: Es war gerade das Verdienst Eugen Vargas, sich dieser Aufgabe unterzogen zu haben. Als erster hat er für die kommunistische Bewegung in scharfer Auseinandersetzung mit dem Opportunismus und bürgerlichen Konzeptionen die Marxschen krisentheoretischen Grundthesen zusammengefaßt.

36 J. Kuczynski, Die Schule Eugen Vargas, in: Studien zu einer Geschichte der Gesellschaftswissenschaften, Bd. 7, a. a. O.;

J. Goldberg, Methodische und theoretische Probleme in Vargas Vierteljahresberichten, in: E. Varga, Wirtschaft und Wirtschaftspolitik, Vierteljahresbericht 1922-1939 (Faksimileausgabe) Bd. 1, a. a. O.

37 L. A. Mendel'son, Teorija i istorija èkonomičeskich krizisov i ciklov, Bd. 1-3, Moskva 1959-1964; I. A. Trachtenberg, Kapitalistische Reproduktion und Wirtschaftskrisen (Theoretischer Abriß), Berlin 1957; F OelBner, Die Wirtschaftskrisen. Erster Band. Die Krisen im vormonopolistischen Kapitalismus, Berlin 1949. 
Wesentliche Bausteine dieser theoretischen Arbeit Eugen Vargas sind in der vorliegenden Ausgabe enthalten. Es handelt sich dabei vor allem um die Schriften „Die Große Krise und ihre politischen Folgen“, „Die Weltwirtschaftskrisen“, „Die kapitalistische Welt vor einer neuen Krise“ und der Abschnitt „Veränderungen im zyklischen Verlauf der Reproduktion nach dem zweiten Weltkrieg“" in den „Beiträgen zu Problemen der politischen Ökonomie des Kapitalismus“. Weitere krisentheoretische Überlegungen Vargas finden sich in den Vierteljahresberichten zur Wirtschaft und Wirtschaftspolitik, z. B. der Abschnitt „Die Krisentheorie von Marx und die Probleme der gegenwärtigen Krise“ im Bericht über das I. Vierteljahr 1931 . $^{38}$

In allen seinen Schriften, die den Problemen der Marxschen Krisentheorie gewidmet sind, geht Eugen Varga konsequent von Marx' Grundthese aus, daß der kapitalistische Grundwiderspruch die Hauptursache der Krisen darstellt. Dabei greift Eugen Varga den bei Marx formulierten Gedanken auf, daß dies nicht nur im Sinne eines allgemeinen Zusammenhanges, nämlich der gesetzmäßigen Unvermeidlichkeit von Überproduktionskrisen im Kapitalismus, zu verstehen ist, sondern daß die Krisen zugleich wesentliche Einschnitte und „Durchgangsstationen“ in der weiteren Entfaltung des kapitalistischen Grundwiderspruchs und damit des historischen Niedergangs des Kapitalismus darstellen. So formuliert Eugen Varga im ersten Teil des Buches „,Die Weltwirtschaftskrisen“ : „. . . der eigentliche Grund für die periodisch wiederkehrenden Krisen und den historisch unvermeidlichen Niedergang des Kapitalismus ist derselbe, nämlich der Widerspruch zwischen dem gesellschaftlichen Charakter der Produktion und der kapitalistischen Form der Aneignung. Die periodisch wiederkehrenden Überproduktionskrisen bedeuten den Ausbruch aller Widersprüche der kapitalistischen Gesellschaftsordnung. Die Hauptursache der Krisen ist der Grundwiderspruch zwischen der gesellschaftlichen Produktion und der privatkapitalistischen Form der Aneignung. Die Krisen stellen eine vorübergehende (,momentane') gewaltsame Lösung der entstandenen scharfen Widersprüche in der erweiterten Reproduktion dar. Doch der Grundwiderspruch des Kapitalismus bleibt nicht nur erhalten, sondern wird mit jeder Krise tiefer und führt unvermeidlich zu einer neuen Überproduktionskrise." ${ }^{39}$ Dieser Ausgangspunkt ermöglichte Eugen Varga, sich konsequent mit den verschiedensten opportunistischen und bürgerlichen Verfälschungen der Marxschen Krisentheorie auseinanderzusetzen und anhand des konkreten Verlaufs der zyklischen Entwicklung der kapitalistischen Wirtschaft immer aufs neue nachzuweisen, $\mathrm{da} ß$ die periodisch wiederkehrenden Überproduktionskrisen unvermeidlich aus der ökonomischen Grundstruktur des kapitalistischen Systems hervorgehen. Zugleich sucht Eugen Varga bei der Analyse der jeweiligen Krise deren Rolle im langfristigen Prozeß der kapitalistischen Wirtschaftsentwicklung zu erfassen.

In dem krisentheoretischen Konzept Eugen Vargas folgt dann die Behandlung der aus dem kapitalistischen Grundwiderspruch erwachsenden inneren Widersprüche der kapitalistischen Produktion, deren Entfaltung unmittelbar zur allgemeinen, relativen Überproduktion führt, die Krise verursacht. Natürlich geht Eugen Varga auch hier von Marx aus. Sein eigenes Verdienst besteht aber darin, die bei Marx an verschiedenen Stellen seines Werkes verstreuten Bemerkungen über die gesetzmäßig zur Krise führenden Widersprüche der kapitalistischen Produktion systematisiert und aufbereitet zu haben. Gestützt auf die Reproduktionsschemata und die Realisationstheorie im zweiten Band des

38 Siehe: Wirtschaft und Wirtschaftspolitik ..., a. a. O., Bd. 4.

39 E. Varga, Die Weltwirtschaftskrisen, in: Bd. 2 dieser Ausgabe, S. 6. 
Werkes „Das Kapital“ zeigt er, wie sich innerhalb des kapitalistischen Reproduktionsprozesses vor allem zwei Widersprüche entfalten: die Disproportionalität zwischen den beiden Abteilungen und den Zweigen sowie der Widerspruch zwischen Produktion und Markt, der sich aus dem Zurückbleiben der Konsumtionskraft der Massen hinter der Produktion ergibt.

Dabei sind es vor allem zwei methodologische Grundfragen, die bei Eugen Varga immer wieder zu Recht in den Vordergrund gestellt werden. Zum einen unterstreicht Eugen Varga den dialektischen Zusammenhang zwischen beiden Widersprüchen. Er bezieht sich dabei auf die Feststellung W. I. Lenins: „Die ,Konsumtionskraft der Gesellschaft und die ,Proportionalität der verschiedenen Produktionszweige‘ sind keineswegs irgendwelche isolierten, selbständigen, nicht miteinander verbundenen Bedingungen. Im Gegenteil, ein bestimmter Stand der Konsumtion ist eines der Elemente der Proportionalität. "40 Eugen Varga beginnt seine Darlegungen daher einmal mit den Reproduktionsschemata und entwickelt im direkten Anschluß das Problem des kapitalistischen Marktes, und ein anderes Mal verfährt er umgekehrt. Varga vermeidet auch eine in manchen krisentheoretischen Arbeiten anzutreffende schematische Zergliederung dieser beiden Widersprüche in eine Vielzahl von „Unter"-Widersprüchen. Eine zweite methodologische Grundfrage ist bei Eugen Varga die Hervorhebung des Unterschiedes zwischen der Kaufkraft und der Konsumtionskraft der Gesellschaft. Bekanntlich setzte sich Karl Marx grundlegend mit dem "Sayschen Theorem“ auseinander, das die Behauptung enthielt, mit der Produktion einer Ware werde auch die entsprechende Kaufkraft produziert, die Summe des Produktenwertes sei gleich der Kaufkraft der Gesellschaft, und folglich sei eine allgemeine Überproduktion nicht möglich. Dieses Theorem geistert noch immer durch die bürgerliche politische Ökonomie. Eugen Varga hat in allen seinen Schriften zur Krisentheorie auf den wesentlichen Unterschied zwischen Kaufkraft und Konsumtionskraft der Gesellschaft hingewiesen. So formuliert er z. B.: „Die Kaufkraft der kapitalistischen Gesellschaft entspricht tatsächlich $c+v+m$, sie ist gleich der Summe des Wertes der in einem Jahr hergestellten Waren. Doch nutzt diese allgemeine Wahrheit wenig, um das Marktproblem zu lösen. Entscheidend ist nicht die Kaufkraft der Gesellschaft, sondern deren Konsumtionsfähigkeit. Unter der Konsumtionsfähigkeit der Gesellschaft versteht Marx jenen Teil der Kaufkraft, den Arbeiter und Kapitalisten für Konsumtionsmittel, für Waren der Abteilung II aufwenden können. Das bestimmt letztlich die Aufnahmefähigkeit des Marktes für alle Arten von Waren, denn die Produktionsmittel werden von den Kapitalisten nur deshalb erworben, um damit Konsumtionsmittel herzustellen." ${ }^{41}$

Eugen Varga zeigt dann auf, da $\beta$ die Konsumtionsfähigkeit der Gesellschaft durch die Kapitalakkumulation relativ, d. h. im Vergleich zum Gesamtwert der hergestellten Waren verringert wird. Je größer der Teil des Kapitals ist, der für die Akkumulation aufgewendet wird, desto geringer ist zwangsläufig der für die Konsumtion bestimmte Teil. Deshalb: „Die Konsumtionsfähigkeit der Gesellschaft entspricht also der Formel: $v+(m-a)$, d. h. der Lohnsumme plus der Mehrwertsumme nach Abzug der Akumulation. ${ }^{\text {“42 }}$ Der durch die Gesetze der Konkurrenz verursachte Zwang des Kapitals zur Akkumulation, zur Beschränkung der Konsumtionskraft der Werktätigen bringt also unausweichlich

40 Siehe: W. I. Lenin, Notiz zur Frage der Theorie der Märkte, in: Werke, Bd. 4, Berlin 1955, S. 48/49.

41 E. Varga, Die kapitalistische Welt vor einer neuen Krise, in: Bd. 2 dieser Ausgabe, S. 400-401.

42 Ebenda, S. 401. 
den Widerspruch zwischen der begrenzten Konsumtionsfähigkeit der Gesellschaft und dem Streben des Kapitals nach unbegrenzter Ausdehnung der Produktion hervor. Dieser Widerspruch, den Marx als „den letzten Grund aller wirklichen Krisen“ bezeichnet, muß unausweichlich zu periodisch wiederkehrenden Überproduktionskrisen führen.

Als nächsten Schritt in der Entwicklung seines krisentheoretischen Konzepts wirft Eugen Varga die Frage auf, warum es bei der Anarchie der Produktion in der kapitalistischen Gesellschaft keine ,,permanenten“ Krisen gibt, warum sich die kapitalistische Wirtschaft in Zyklen entwickelt. Hier entwickelt Eugen Varga, wie die kapitalistische Akkumulation zunächst über die Schranken des Marktes hinaustreibt, ohne daß die Überproduktion bereits sichtbar wird und Realisierungsschwierigkeiten auftauchen. Es vollzieht sich eine „Produktion für die Produktion“. Da diese aber letztlich von der individuellen Konsumtion abhängt, tritt infolge der begrenzten Konsumtionskraft der Massen schließlich Überproduktion auf, bricht die Krise aus. Der kapitalistische Akkumulationsprozeß ist also die Ursache für ein zeitweiliges schrankenloses Hinaustreiben über die Grenzen der Konsumtionsfähigkeit, das sich in zyklischer Form, in einzelnen Phasen eines Zyklus vollzieht. Eugen Varga hat in diesem Zusammenhang auch einen selbständigen Beitrag zur Einteilung und Charakterisierung der einzelnen Zyklusphasen geleistet. So versucht er bei der Analyse der konkreten Wirtschaftslage immer, bestimmte Kriterien für die einzelnen Phasen Krise, Depression, Belebung, Aufschwung und Prosperität herauszuarbeiten. Methodologisch ist in diesem Zusammenhang besonders hervorzuheben, daß Varga konsequent von der Krise als der konstituierenden Phase des Zyklus ausgeht. Er hebt damit die Marxsche These hervor, daß die zyklischen Krisen eine wesentliche Rolle im Funktionsmechanismus der kapitalistischen Ökonomik spielen, da sie durch die „momentane“ Lösung der inneren Widersprüche der kapitalistischen Produktion die Grundlage für einen neuen Aufschwung bereiten und in ihrer periodischen Wiederholung damit auch die hauptsächliche Bedingung für die erweiterte Reproduktion des Kapitalismus überhaupt darstellen. Bekanntlich setzt die heutige marxistische Krisenforschung gerade an dieser These an, inwieweit diese Funktion der Krisen unter staatsmonopolistischen Bedingungen noch voll wirken kann, inwieweit andere Krisenprozesse solche Funktionen in allen bzw. mehreren Phasen des Zyklus ausüben und welche Modifikationen des Zyklusverlaufs daraus erwachsen. Von besonderem Interesse sind Eugen Vargas Untersuchungen über die Depressionsphase, die er anläßlich der „Großen Krise“ von 1929-1933 anstellte. ${ }^{43}$ Die Aktualität dieser Fragestellungen zeigte sich erst unlängst in der Diskussion über die Einschätzung der kapitalistischen Wirtschaftsentwicklung nach der Weltwirtschaftskrise von 1974-1975. Eine weitere wichtige Frage, die Eugen Varga bei seiner Darlegung der Marxschen Krisentheorie behandelt, ist die Periodizität der Krisen. Ausgehend von Marx, der den Umschlagszyklus des fixen Kapitals als die materielle Basis des zyklischen Ganges der kapitalistischen Reproduktion nachwies, sucht Eugen Varga in seiner Analyse des Zyklusverlaufes während der allgemeinen Krise des Kapitalismus die neuen Tendenzen im Umschlag des fixen Kapitals herauszuarbeiten.

Dies alles sind Hauptelemente einer Systematisierung der Marxschen Krisentheorie, die bis heute zum methodologischen Rüstzeug der marxistischen Krisenforscher gehören. Wenn auch in den einzelnen Arbeiten marxistischer Politökonomen dem einen oder anderen Aspekt ein größeres Gewicht beigemessen wird und auch in verschiedenen Fragen

43 E. Varga, Vierteljahresbericht $\mathrm{I} / 1934$, in: Wirtschaft und Wirtschaftspolitik ..., a. a. O., Bd. 5 . 
unterschiedliche Auffassungen bestehen, so bleibt es das Verdienst Eugen Vargas, erstmalig den umfassenden Versuch unternommen zu haben, die Marxschen Thesen über die zyklischen Krisen in ein systematisches Konzept gebracht zu haben.

Wie bereits erwähnt, erwarb sich Eugen Varga besondere Verdienste um die konkrete Anwendung der Marxschen Krisentheorie auf die Analyse der aktuellen Wirtschaftsprozesse im Kapitalismus. Eugen Varga hat es wie kaum ein anderer meisterhaft verstanden, das Faktenmaterial zu analysieren, um Kriterien für den erreichten Stand des jeweiligen Wirtschaftszyklus herauszufinden. Sein besonderes Augenmerk richtete sich auf Daten über den Akkumulationsprozeß, den Widerspruch zwischen Produktion und Markt, die Bewegung der Aktienkurse und Neuemissionen, die Preisentwicklung und andere Hauptkennziffern der Wirtschaftsentwicklung. Es gelang Eugen Varga, selbst mit den lückenhaften Angaben, die ihm damals zur Verfügung standen, rechtzeitig und vorausschauend Veränderungen im ökonomischen Zyklus des Kapitalismus zu erkennen und vorausschauende Aussagen für dessen weiteren Verlauf zu treffen. Wir können uns hier mit diesem Tätigkeitsfeld Eugen Vargas nicht näher beschäftigen und verweisen auf den interessanten Aufsatz von Jörg Goldberg in der mehrfach erwähnten Neuausgabe der Vargaschen Vierteljahresberichte, in welchem der Anwendung der Marxschen Krisentheorie durch Eugen Varga auf die konkrete Wirtschaftsanalyse besonderer Raum gegeben wird. ${ }^{44}$

Einen eigenständigen Beitrag leistete Eugen Varga auch bei der Analyse der Agrarkrise des Kapitalismus. Diese Frage, die in der Forschungsarbeit und in der wissenschaftlichen Diskussion unter den marxistisch-leninistischen Ökonomen in den letzten Jahren in den Hintergrund getreten ist, nimmt in den krisentheoretischen Arbeiten Eugen Vargas einen besonderen Platz ein. Es ist sicherlich eine Aufgabe der politökonomischen Forschung, sich dieser Problematik erneut anzunehmen und dabei die wertvollen Hinweise Eugen Vargas auszuwerten.

Gleiches gilt für seine Beiträge zu den Teilkrisen und strukturellen Krisen der kapitalistischen Wirtschaft. So finden sich in den Vierteljahresberichten wichtige methodologische Auffassungen Eugen Vargas, die er anläßlich der Zwischenkrisen 1924 und 1927 in den USA formulierte. Außerdem beschäftigte er sich eingehend mit der Finanzkrise, mit den Preisbewegungen auf dem kapitalistischen Weltmarkt und mit strukturellen Krisen in einzelnen Wirtschaftszweigen. Gerade diese Arbeiten sind für die Analyse des ökonomischen Zyklus in der gegenwärtigen Entwicklungsetappe des Kapitalismus von ganz besonderer Bedeutung. Sie weisen uns auf Momente hin, die bisher in der theoretischen Forschung nicht genügend ausgewertet wurden. ${ }^{45}$ Schließlich leistete Eugen Varga einen entscheidenden Beitrag zur Erforschung der Geschichte der Wirtschaftskrisen. Von ihm wie auch von seinen Mitarbeitern stammen zahlreiche Arbeiten über die Entwicklung der Krisen im Kapitalismus seit 1825. Besonders zu erwähnen sind hierbei das Werk Eugen Vargas „Die Weltwirtschaftskrisen 1857-1938“" sowie die Arbeiten von L. A. Mendel'son über Theorie und Geschichte der Krisen im Kapitalismus.

Auf eine Frage soll schließlich noch näher eingegangen werden, weil sie für die heutige Forschung einen besonderen Stellenwert besitzt: die Darstellung des Zusammenhanges zwischen allgemeiner Krise und zyklischer Krise in den Arbeiten Eugen Vargas.

44 Siehe Fußnote 36, S. XXIX.

45 Die „Vierteljahresberichte" sind gerade in dieser Hinsicht eine wahre Fundgrube. Die meisten Beiträge finden sich in den Berichten von 1925-1932 (Bd. 3 und 4 der fünfbändigen Faksimileausgabe). 
Seit den 20er Jahren stehen im Zentrum von Vargas Untersuchungen über die ökonomischen Merkmale der allgemeinen Krise des Kapitalismus vor allem die Veränderungen in der zyklischen Bewegung und in den zyklischen Krisen der kapitalistischen Wirtschaft, nämlich ihre Tendenz zu chronischer Schärfe und zur Deformierung des Phasenablaufs. Die realen Prozesse, so die Wirtschaftskrise von 1920, die komplizierte Veränderung der ökonomischen Situation in der ,relativen Stabilisierung “ und dann vor allem die tiefe Krise von 1929 bis 1933 und die ihr folgende lange Depressionsphase waren die Ursache dafür, da $B$ Varga einen großen Teil seiner Aufmerksamkeit eben auf die Wechselwirkung zwischen zyklischer Bewegung und allgemeiner Krise konzentrierte und damit den entscheidenden Grundstock für das Erkennen der ökonomischen Züge der allgemeinen Krise - speziell in ihrer ersten Etappe - legte.

Für Eugen Varga zeigt sich diese Wechselwirkung darin, da $\beta$ die Schärfe der zyklischen Krisen der Tatsache geschuldet ist, da $\beta$ sich die zyklische Entwicklung auf der Basis der allgemeinen Krise des Kapitalismus vollzieht, wobei die zyklischen Krisen wiederum die Vertiefung der allgemeinen Krise weiter vorantreiben. Zu dieser Auffassung gelangte Eugen Varga vor allem durch die Analyse der Krise von 1929-1933, der ,ersten Weltkrise“ "nach dem ersten Weltkrieg, in der Periode der allgemeinen Krise. Er schrieb: „Diese ihre historische Stelle bestimmt auch ihre konkreten Besonderheiten. Sie ist zwar zweifellos eine zyklische Überproduktionskrise, aber keinesfalls eine ,normale' Krise, ,keine einfache Wiederholung der vorhergehenden Krisen'. Ihr Verlauf wurde stark modifiziert durch den Einfluß der allgemeinen Krise des Kapitalismus, auf deren Basis sie abläuft ...46 Diese Krise war „tiefer, länger und in jeder Beziehung verheerender" als irgendeine der früheren zyklischen Krisen und hat, wie Varga hervorhebt, durch die „tiefgehende Erschütterung des ganzen kapitalistischen Systems eine neue, höhere Stufe der allgemeinen Krise des Kapitalismus geschaffen ..."47.

Diese Wechselwirkung - oder wie Varga auch schon 1932 formuliert: „Die Verflechtung der allgemeinen Krise des Kapitalismus mit der zyklischen Krise'48 - wird auch bei der Analyse der folgenden Krisen, so vor allem der Krise von 1937/1938, hervorgehoben und als eine neue Grundtendenz herausgestellt.

Welches sind die Hauptfaktoren der allgemeinen Krise, die auf den Zyklus einwirken? In dem Buch „Die Weltwirtschaftskrisen“ gibt Eugen Varga eine verallgemeinernde Zusammenfassung. Es sind vor allem: die Verschärfung des Marktproblems, der chronische Überfluß an fixem Kapital, chronische Massenarbeitslosigkeit und rasch fortschreitende Verelendung der Arbeiterklasse sowie die Verflechtung der zyklischen Krisen mit der chronischen Agrarkrise. Zugleich verweist Varga auf den krisenverschärfenden Einfluß der Monopole (weitere Einschränkung der Konsumtionskraft der Gesellschaft; Rolle der Monopolherrschaft, besonders der Monopolpreise, die den Ausweg aus der Krise erschweren), der bereits seit der Anfangsperiode des Imperialismus vorhanden ist. ${ }^{49}$

Eine besondere Rolle spielt bei dieser Einschätzung die Verschärfung des Marktproblems. Varga unterstreicht hier vor allem zwei Aspekte: die zunehmende relative Einschränkung der Konsumtionskraft der Gesellschaft und die chronische Nichtauslastung der Produktionskapazitäten, die die Reproduktion des fixen Kapitals erschwert. Für beide Aspekte

46 E. Varga, Vierteljahresbericht IV/1933, in: Wirtschaft und Wirtschaftspolitik ..., a. a. O., Bd. 5.

47 Ebenda.

48 E. Varga, Vierteljahresbericht III/1932, in: Wirtschaft und Wirtschaftspolitik ..., a. a. O., Bd. 4.

49 E. Varga, Die Weltwirtschaftskrisen, in: Bd. 2 dieser Ausgabe, S. 25. 
nennt Varga als Ursachen: die historische Tendenz des immer stärkeren Zurückbleibens der Konsumtionskraft der Gesellschaft hinter der Produktion (,relative Einengungstendenz des kapitalistischen Marktes") und in diesem Zusammenhang die den Markt einengende Wirkung der Monopole sowie als ,äußeren Faktor“ die Einengung des kapitalistischen Marktes durch das Bestehen der Sowjetunion. ${ }^{50}$ „Die Aufnahmefähigkeit des Marktes ... (ist) ... chronisch - nicht nur in der Krise und Depressionsphase des Zyklus - zu gering ...., um den Absatz der Waren bei voller Ausnutzung der vorhandenen Produktionskräfte zu ermöglichen". 51 Für den Krisenzyklus ergeben sich im Ergebnis der Herrschaft der Monopole einschneidende Wirkungen durch die Veränderungen im Prozeß der Reproduktion des fixen Kapitals. „Im monopolistischen Kapitalismus reißen die Monopolisten einen wachsenden Teil des Gesamtprofites an sich, akkumulieren Riesensummen, aber die Notwendigkeit, das Angebot der Monopolware in entsprechend engen Grenzen zu halten, ist ein schweres Hindernis der produktiven Anlage des neuakkumulierten Kapitals. Die Möglichkeit der Ausdehnung des kapitalistischen Marktes durch ,Produktion für die Produktion', auf die Lenin so großes Gewicht gelegt hatte, wird durch die Monopole stark eingeengt!"52 Gerade in der Verschärfung des Marktproblems, besonders in den Veränderungen der Reproduktion des fixen Kapitals, sieht Eugen Varga eine wesentliche Ursache für die Modifikation des Ganges der zyklischen Entwicklung in der allgemeinen Krise des Kapitalismus. „Das bereits vorhandene, nicht ausgenutzte fixe Kapital wird zu einem Hemmnis für die Erweiterung des fixen Kapitals. Dieser Umstand deformiert den Verlauf des Zyklus in der Periode der allgemeinen Krise des Kapitalismus noch mehr: Die Phasen der Krise und der Depression dauern länger und sind tiefer, während die Phasen der Belebung und des Aufschwungs kürzer und weniger umfassend sind." 53 Diese Verallgemeinerung seiner Analyse der Modifikation des Krisenzyklus hat Eugen Varga an vielen Stellen wiederholt und präzisiert.

Bevor wir auf einige Gesichtspunkte der Vargaschen Analyse des Zyklus nach dem zweiten Weltkrieg eingehen, müssen noch zwei wichtige methodologische Fragen erwähnt werden. Eugen Varga ging auch bei der Einschätzung der Modifikationen des Krisenzyklus in der allgemeinen Krise von der Funktion der Krisen im Mechanismus der kapitalistischen Reproduktion aus. Der allgemeine Ausdruck dieser Modifikation ist eben die veränderte Funktion der Krisen bei der zeitweiligen Lösung der inneren Widersprüche der kapitalistischen Produktion. „Unter dem Einfluß der allgemeinen Krise des Kapitalismus kann dieser Prozeß seine Funktion - die gewaltsame, vorübergehende Lösung der Widersprüche - nicht wie früher erfüllen." ${ }^{\text {(54 }}$ Dieser methodologische Ansatz ist gerade für die heutige Krisenanalyse von Bedeutung, da sich hier sofort die Frage anschließt, welche anderen Krisenprozesse neben der zyklischen Krise unter staatsmonopolistischen Bedin-

50 E. Varga, Vierteljahresbericht II/1933, in: Wirtschaft und Wirtschaftspolitik ..., a. a. O., Bd. 5.

51 Ebenda.

52 Ebenda. An einigen Stellen verweist Eugen Varga auch darauf, daß die großen Monopole ,eine gewisse Möglichkeit" haben, die Produktion einzuschränken, bevor sich große unverkäufliche Vorräte bei ihnen anhäufen und die Krisen ,in ihrem Charakter insofern modifiziert werden können “..$^{50}$ Allerdings ist dieser Gedanke von Varga später bei seinen Untersuchungen über den Widerspruch zwischen Produktion und Markt nicht weiter entwickelt worden. Siehe hierzu u. a.: Vierteljahresberichte III/1929 und I/1931, in: Wirtschaft und Wirtschaftspolitik ..., a. a. O., Bd. 4.

53 E. Varga, Die kapitalistische Welt vor einer neuen Krise, in: Bd. 2 dieser Ausgabe, S. 407.

54 E. Varga, Vierteljahresbericht III/1932, in: Wirtschaft und Wirtschaftspolitik ..., a. a. O., Bd. 4. 
gungen „Krisenfunktionen“ im zyklischen Gang der kapitalistischen Wirtschaftsentwicklung ausüben, dadurch die Kapitalverwertung beeinflussen und den Zyklus weiter modifizieren können. Eugen Varga ist auf diese Fragen auch in späteren Schriften leider nur wenig eingegangen.

Zweitens sind in methodologischer Hinsicht seine Untersuchungen zur Synchronität des Krisenzyklus im kapitalistischen System von besonderem Interesse. Varga ist dieser Frage bereits vor der Krise von 1929-1933 nachgegangen. Er hat dabei stets den Zusammenhang zwischen der Ungleichmäßigkeit der ökonomischen und politischen Entwicklung der kapitalistischen Länder und dem Verlauf des Krisenzyklus in ihnen hervorgehoben. Er sagte am Vorabend der "Großen Krise“ voraus, daß sich die Synchronität in einer umfassenden Weltwirtschaftskrise herstellen wird, wies aber zugleich nach Ausbruch dieser Krise darauf hin, daß diese Synchronisierung nicht von Dauer sein muß. „Erstreckt sich die Krisenphase auf die ganze kapitalistische Welt, so ist dies keinesfalls ein Beweis, daß auch ein einheitlicher Aufschwung und Hochkonjunktur für alle Länder erfolgen wird! . . . Wir glauben im Gegenteil, daß zwar die gegenwärtige Krisenphase selbstverständlich nach einer gewissen Zeit in die Depression übergehen, daß aber ein großer Teil der kapitalistischen Welt zu keiner Hochkonjunktur gelangen wird; daß sich die Einheitlichkeit der kapitalistischen Weltwirtschaft wohl in gemeinsamen Krisenphasen, nicht aber in einer gemeinsamen Hochkonjunkturphase dokumentieren wird."5s Varga hat mit dieser Einschätzung recht behalten und zugleich in seiner Beweisführung wichtige Hinweise für die heutige Analyse der Synchronität des Krisenzyklus gegeben.

Eugen Varga leistete also eine bedeutende Pionierarbeit bei der Aufdeckung der Verflechtung der allgemeinen Krise des Kapitalismus mit den zyklischen Krisen. Das gilt vor allem für die grundlegende Arbeit bei der umfassenden Einschätzung der ökonomischen Widersprüche und für seine Voraussagen über den weiteren Verlauf des Zyklus speziell in der ersten Etappe der allgemeinen Krise des Kapitalismus.

Dieser Hinweis erscheint uns wesentlich, weil z. B. in der zweiten Etappe der allgemeinen Krise diese ökonomischen Widersprüche des Krisenzyklus nicht die bestimmende Rolle für die Entfaltung der allgemeinen Krise spielten. Hier muß man vielmehr die Auswirkungen des Entstehens eines sozialistischen Weltsystems und des beginnenden Zerfalls des Kolonialsystems auf die kapitalistische Weltwirtschaft, die Nachkriegszerrüttung der Wirtschaft vor allem in Westeuropa, die neue Rolle und weltweite Expansion der USA als imperialistischer Hauptmacht, die Einflüsse der staatsmonopolistischen Regulierung usw. in den Vordergrund rücken. Natürlich spielten auch die erneute Entfaltung der Widersprüche zwischen Produktion und Markt, vor allem die starke Asynchronität des Krisenzyklus eine Rolle, aber eben mit einem anderen Gewicht in der Gesamtheit der Faktoren. Mit der Entfaltung der dritten Etappe der allgemeinen Krise des Kapitalismus hat vor allem seit Mitte der 70er Jahre die Verflechtung von allgemeiner Krise und zyklischer Entwicklung wieder besonders deutliche Züge angenommen, was auch die von Varga hervorgehobenen Erscheinungen des Kapitalüberflusses und der Massenarbeitslosigkeit erneut in den Vordergrund der ökonomischen Erscheinungen rückt. Gleichzeitig unterscheiden sich jedoch das Gesamtbild des Kapitalismus, die Verflechtung der verschiedenen Faktoren, der Einfluß der revolutionären Veränderungen in der Welt beträchtlich von den Bedingungen der ersten und zweiten Etappe der allgemeinen Krise. Auch der Stellenwert der für die zyklische Entwicklung und Krise verantwortlichen

55 E. Varga, Vierteljahresbericht I/1930, in: Wirtschaft und Wirtschaftspolitik ..., a. a. O., Bd. 4. 
Widersprüche, die Verknüpfung der Ursachen, die heute dem Kapitalüberfluß und der Arbeitslosigkeit zugrunde liegen, ihr Ausma $\beta$ und ihre Wirkungen gilt es sehr konkret für die Gegenwart zu untersuchen, wenn wir im Sinne Vargas die Theorie der allgemeinen Krise des Kapitalismus auf die Bedingungen ihrer dritten Etappe anwenden wollen.

Nach dem zweiten Weltkrieg beschäftigte sich Varga mit den Besonderheiten des zyklischen Verlaufs der Reproduktion in den 50er Jahren und Anfang der 60er Jahre. Er stellte bestimmte Veränderungen der zyklischen Bewegung im Vergleich zur Periode zwischen den beiden Weltkriegen fest, vor allem das Auseinanderfallen des zyklischen Rhythmus in den USA einerseits und in Westeuropa anderseits. Für die weitere Entwicklung sagte Varga die Verstärkung der Tendenz zur Verkürzung des Zyklus und zur Vertiefung der Krisen voraus. ${ }^{56}$ „Die Wirtschaftskrisen werden tiefer sein, als sie in den ersten 15 Jahren nach dem zweiten Weltkrieg waren", lautete Vargas Prognose in seiner Schrift „Der Kapitalismus des zwanzigsten Jahrhunderts“57, und seine Voraussage hat sich - wenn auch mit einiger Verzögerung - in den 70er Jahren eindeutig erfüllt.

In den „Beiträgen zu Problemen der politischen Ökonomie des Kapitalismus“ hat Varga einige seiner Grundthesen aus den 20er und 30er Jahren wieder aufgegriffen und um neue Momente bereichert. So bezieht er bei der Analyse der Reproduktion des fixen Kapitals solche Faktoren wie den beschleunigten wissenschaftlich-technischen Fortschritt und die Rolle des Staates bei der Erneuerung des fixen Kapitals ein. Zusammenfassend kommt Varga dann zu der Schlußfolgerung, daß die Voraussetzungen für eine Überproduktionskrise beschleunigt heranreifen, der Zyklus verkürzt wird, weil infolge der nichtausgelasteten Kapazitäten mit denselben Investmitteln eine weit größere Erweiterung der Kapazitäten erreicht wird als beim Bau neuer Betriebe und sich der Zeitraum verringert, innerhalb dessen die Neuinvestitionen produktionswirksam werden. ${ }^{58}$ Diese These spielt, wie auch andere Thesen Eugen Vargas - so zum Marktproblem in der Periode nach dem zweiten Weltkrieg - , in der gegenwärtigen Diskussion der marxistischen Krisenforscher nach wie vor eine wichtige Rolle.

Die neuesten Entwicklungstendenzen zeigen, daß die dritte Etappe der allgemeinen Krise dem Krisenzyklus ihren spezifischen Stempel aufdrückt und wir nunmehr vor der Aufgabe stehen, die neuen Tatsachen und Tendenzen sorgfältig zu studieren und Schlußfolgerungen für die weitere Perspektive abzuleiten. Dabei geben uns die Arbeiten Eugen Vargas wesentliche Anregungen.

Angesichts des reichen wissenschaftlichen Werkes von Eugen Varga ist es eine schwierige Aufgabe, eine repräsentative Auswahl zusammenzustellen. Die vorliegende 3bändige Ausgabe unternimmt den Versuch, einen möglichst umfassenden Einblick in das Schaffen Eugen Vargas über fünf Jahrzehnte hinweg zu geben. Als Grundlage diente die 3bändige Ausgabe, die 1974 im Moskauer Verlag „Nauka“ erschienen ist. ${ }^{59}$ Die vorliegende Ausgabe

56 E. Varga, Beiträge zu Problemen der politischen Ökonomie des Kapitalismus, in: Bd. 3 dieser Ausgabe, S. $248-272$.

57 E. Varga, Der Kapitalismus des zwanzigsten Jahrhunderts, in: Bd. 3 dieser Ausgabe, S. 99.

58 E. Varga, Beiträge zu Problemen der politischen Ökonomie des Kapitalismus, in: Bd. 3 dieser Ausgabe, S. 269.

59 E. S. Varga, Izbrannye proizvedenija, Bd. 1-3, Moskva 1974, izdatel'stvo „Nauka“. 
geht jedoch über die in der UdSSR veröffentlichte Fassung hinaus, da für den Leserkreis in der Deutschen Demokratischen Republik weitere Arbeiten aufgenommen wurden. Dabei handelt es sich vor allem um solche Schriften, die von besonderer Aktualität für die heutige Imperialismusanalyse sind bzw. die bisher nicht in deutscher Sprache vorgelegen haben.

Die bereits erwähnten Vierteljahresberichte über Wirtschaft und Wirtschaftspolitik (1922-1939) konnten in dieser Ausgabe nur unter einigen Aspekten berücksichtigt werden. Dabei kann darauf verwiesen werden, daß diese Vierteljahresberichte im Jahre 1977 im Verlag „Das europäische Buch“, Westberlin, in einer fünfbändigen Faksimileausgabe, vollständig neu herausgegeben wurden. ${ }^{60}$ Diese verdienstvolle Buchausgabe ist zweifellos eine wesentliche Ergänzung der hiermit vorgelegten dreibändigen Ausgabe der ausgewählten Schriften Eugen Vargas.

Die Dreiteilung der Bände - „Der Beginn der allgemeinen Krise des Kapitalismus“, „Die Wirtschaftskrisen“, ,Der Kapitalismus nach dem zweiten Weltkrieg“ - widerspiegelt gleichermaßen die theoretischen Schwerpunkte wie auch die Entwicklungsetappen des wissenschaftlichen Lebenswerks Eugen Vargas.

Der erste Band beginnt mit der Schrift „Das Geld: Seine Herrschaft in Friedenszeiten und sein Zusammenbruch während des Krieges". Es handelt sich hier um die erste größere theoretische Arbeit Eugen Vargas, die bereits zu wesentlichen Teilen im Jahre 1914 fertiggestellt war und nach ergänzenden Analysen über die Kriegswirtschaft im Jahre 1918 erschien. Sie ist eine glänzende Darstellung der Marxschen Geldtheorie in ihrer Anwendung auf die konkreten Verhältnisse in Ungarn und in anderen kapitalistischen Ländern. Diese Arbeit erscheint erstmalig in deutscher Sprache. Sie kann in ihren theoretisch-methodologischen Ansätzen als ein wichtiger Beitrag auch zur Analyse der heutigen Entwicklung des Geldes und der Inflation im Kapitalismus gelten. Es folgt dann die bereits erwähnte Arbeit Eugen Vargas „Die wirtschaftspolitischen Probleme der proletarischen Diktatur“, die erstmalig in deutscher Sprache im Jahre 1920 erschien und in mehrere Sprachen übersetzt wurde. Der Verfasser schrieb die Arbeit während der Emigration unmittelbar nach der Niederlage der Ungarischen Räterepublik. W. I. Lenin hat diese Arbeit hoch eingeschätzt, dabei zugleich auch einzelne ihrer Aussagen kritisiert. Auf dem zweiten Kongreß der Kommunistischen Internationale bezog sich Lenin auf diese Schrift Eugen Vargas, als er die falschen Thesen Crispiens über die Politik gegenüber den Kleinbauern kritisierte. ${ }^{61}$

Die Arbeiten „Die Krise der kapitalistischen Weltwirtschaft“ (1922) und „Die Niedergangsperiode des Kapitalismus“ (1923) sind gleichermaßen interessant für die Einschätzung des zyklischen Verlaufs der kapitalistischen Wirtschaft nach dem ersten Weltkrieg wie auch für die Herausarbeitung der ersten Thesen Eugen Vargas zur Theorie der allgemeinen Krise des Kapitalismus. In den ersten Band wurde außerdem einer der Vieteljahresberichte Eugen Vargas aufgenommen. Es handelt sich hierbei um den Bericht über das vierte Vierteljahr 1929, in dem Eugen Varga seine berühmte Prognose über den Verlauf der Weltwirtschaftskrise von 1929-1933 formulierte. An diesem Beispiel soll auch verdeutlicht werden, wie Eugen Varga in jedem dieser Berichte versuchte, drei wesentliche Elemente miteinander

60 E. Varga, Wirtschaft und Wirtschaftspolitik, Vierteljahresberichte 1922-1939 (Faksimileausgabe Bd. 1-5), hg. von J. Goldberg, Berlin (West) 1977.

61 W. I. Lenin, II. Kongre $B$ der Kommunistischen Internationale, Rede über die Bedingungen für die Aufnahme in die Kommunistische Internationale, 30. Juli 1920, in: Werke, Bd. 31, Berlin 1959, S. 237. 
zu verbinden: eine Einschätzung der aktuellen Wirtschaftsentwicklung mit Voraussagen über deren weiteren Verlauf, Beiträge zu einem ausgewählten theoretischen Problem sowie kurze Länderanalysen. Als letzter Beitrag findet sich im ersten Band die Schrift Eugen Vargas „Die historischen Wurzeln der Besonderheiten des deutschen Imperialismus“. Sie wurde 1943 geschrieben und in deutscher Sprache 1946 veröffentlicht. Die besondere Bedeutung dieser Arbeit ergibt sich daraus, da $\beta$ sie eine der ersten marxistischen Analysen der Entwicklung des deutschen Imperialismus war, die nach dem zweiten Weltkrieg einen großen Leserkreis fand und viele junge marxistische Wissenschaftler in unserer Republik über die Grundzusammenhänge der Entwicklung des deutschen Imperialismus aufgeklärt hat.

Im zweiten Band sind vor allem Arbeiten Eugen Vargas zur Einschätzung der zyklischen Krisen des Kapitalismus enthalten. Als erstes sind die theoretischen Kapitel des großen Sammelbandes über „Die Weltwirtschaftskrisen“ abgedruckt. Der Band wurde 1937 von Eugen Varga unter der Mitwirkung zahlreicher Mitarbeiter des damaligen Instituts für Weltwirtschaft und Weltpolitik herausgegeben. Diese Abschnitte enthalten eine Darlegung der Marxschen Krisentheorie, ergänzt um neue theoretische Verallgemeinerungen Vargas, sowie eine kurze Darstellung des Verlaufs der zyklischen Krisen seit ihrem Beginn im Jahre 1825 .

Es folgt dann der Beitrag von Eugen Varga „Konjunkturforschung und Krisentheorie“, der in einem der Vierteljahresberichte im Jahre 1927 abgedruckt wurde. Diese Schrift ist unter zwei Gesichtspunkten von besonderem Interesse. Zum einen stellt sie eine Auseinandersetzung mit der bürgerlichen Konjunkturforschung dar, und zugleich gibt sie wertvolle Hinweise für die marxistischen Wissenschaftler, die sich mit der Analyse des zyklischen Verlaufs der kapitalistischen Wirtschaft beschäftigen. Dies gilt insbesondere für die Methodik der Ausnutzung der bürgerlichen Statistik und der Daten und Fakten der bürgerlichen Presse überhaupt.

Im dritten Teil des zweiten Bandes sind einige Studien über die Entwicklung einzelner imperialistischer Hauptländer zusammengefaßt. Diese Auszüge über die Entwicklung Englands, Deutschlands, Frankreichs, der USA, Italiens und Japans sollen verdeutlichen, nach welcher Methode Eugen Varga die Länderanalysen anfertigte, und wie er es verstand, historische Wurzeln der Besonderheiten der ökonomischen Entwicklung in den einzelnen Ländern, langfristige Entwicklungstendenzen, einschließlich des Einflusses wesentlicher politischer Faktoren, mit der Einschätzung des aktuellen Ganges der kapitalistischen Wirtschaft zu verbinden. Sie geben somit gleichermaßen Aufschluß über die marxistische Analyse der damaligen Situation in den einzelnen kapitalistischen Ländern wie auch für die Methode einer Länderanalyse unter den heutigen Bedingungen. Es folgen dann zwei Arbeiten über die große Krise von 1929-1933.

Im vierten Teil des Bandes sind die ersten fünf Kapitel des berühmten Buches Eugen Vargas „Die Große Krise und ihre politischen Folgen. Wirtschaft und Politik 1928-1934" abgedruckt. In diesem Hauptteil des Vargaschen Werkes finden sich die Grundaussagen Eugen Vargas über die Ursachen der besonderen Tiefe der Weltwirtschaftskrise von 1929-1933 sowie seine Einschätzung der erfolglosen Bemühungen der Bourgeoisie zur ,künstlichen“ Überwindung der Krise. In engem Zusammenhang damit ist die zweite Arbeit aus dieser Periode „Neue Erscheinungen während der Weltwirtschaftskrise“ anzusehen. Sie wurde erstmalig im Jahre 1934 veröffentlicht und ergänzt die vorher genannte Schrift hinsichtlich der Einschätzung der neuen Tendenzen des zyklischen Verlaufs unter den Bedingungen der allgemeinen Krise des Kapitalismus. Es folgt dann die Arbeit Vargas „Die kapitalistische 
Welt vor einer neuen Krise" aus dem Jahre 1938. In ihr ist die Voraussage Eugen Vargas enthalten, daß die kapitalistische Wirtschaft unausweichlich einer neuen Weltwirtschaftskrise entgegengeht, wenn nicht der Ausbruch eines neuen Weltkrieges diese Krise deformiert. Die beiden letztgenannten Schriften erscheinen erstmals vollständig in deutscher Sprache.

Als letzte Arbeit im zweiten Band finden sich einzelne Auszüge aus der umstrittenen Arbeit Eugen Vargas „Veränderungen in der Wirtschaft des Kapitalismus im Ergebnis des zweiten Weltkrieges“. Die bereits erwähnte „Varga-Diskussion“" wurde seinerzeit auch in unserer Republik als Beilage zur "Sowjetwissenschaft" veröffentlicht. ${ }^{62}$ Eugen Varga hat sich später von einigen der in dieser Arbeit enthaltenen Thesen distanziert. Trotzdem ist gerade diese Arbeit, die unmittelbar nach Beendigung des zweiten Weltkrieges geschrieben wurde, als Beitrag für den wissenschaftlichen Meinungsstreit über grundlegende Entwicklungstendenzen des Kapitalismus auch und gerade heute von besonderem Interesse. Ausschnitte aus diesem Buch sind bisher in deutscher Sprache noch nicht erschienen. Die Herausgeber folgten bei der Auswahl der Kapitel aus diesem Buch der dreibändigen sowjetischen Ausgabe.

Als letzte Arbeit enthält der zweite Band die Schrift Eugen Vargas „Das ,Kapital " von Karl Marx und der moderne Kapitalismus“, die im Jahre 1962 geschrieben wurde. In ihr gibt Eugen Varga wichtige Hinweise für die Methodik der Auseinandersetzung mit der bürgerlichen Wissenschaft und die aktuelle Anwendung der Marxschen Grundthesen.

Der dritte Band enthält zwei bedeutende Arbeiten Eugen Vargas aus seiner letzten Schaffensperiode. Es handelt sich einmal um die Arbeit „Der Kapitalismus des zwanzigsten Jahrhunderts", die im Jahre 1961 fertiggestellt wurde und 1962 in unserer Republik in deutscher Sprache erschien. Diese Arbeit hat bis heute ihre große Aktualität bewahrt. Eugen Varga gibt in ihr einen Überblick über Haupttendenzen der kapitalistischen Entwicklung seit dem Beginn unseres Jahrhunderts. Es ist die umfassendste und reifste Einschätzung, die Eugen Varga über den Verlauf und die Etappen der allgemeinen Krise des Kapitalismus hinterlassen hat. Manche seiner prognostischen Einschätzungen im letzten Teil der Arbeit sind bis heute umstritten. Sie zeugen, wie auch die früheren Arbeiten Eugen Vargas, von dem wissenschaftlichen Streben des Verfassers, langfristige Entwicklungstendenzen des Kapitalismus zu erfassen, und von seinem Mut zu prognostischen Aussagen.

Die zweite Arbeit bildet der Sammelband „Beiträge zur Problemen der politischen Ökonomie des Kapitalismus“, der im Jahre 1964 veröffentlicht wurde. In dieser Arbeit nimmt Eugen Varga zu vielen politökonomischen Fragen Stellung, die in seinem gesamten wissenschaftlichen Lebensweg in der Diskussion eine große Rolle gespielt haben. Das Spektrum der in dieser Schrift enthaltenen Fragen ist außerordentlich breit und reicht von einer Abhandlung über das ökonomische Grundgesetz des Kapitalismus, eine Frage, die gerade im Zusammenhang mit der Arbeit J. W. Stalins „Ökonomische Probleme des Sozialismus in der UdSSR" heftig diskutiert wurde, bis zu dem Problem der asiatischen Produktionsweise, das in den Arbeiten Eugen Vargas in den zwanziger Jahren und zu Beginn der dreißiger Jahre eine große Rolle gespielt und zu heftigem Meinungsstreit geführt hatte. Eugen Varga setzt sich in allen von ihm behandelten Fragen leidenschaftlich mit dogmatischen Entstellungen auseinander, orientiert auf die Analyse der neuen Entwicklungs-

62 Diskussion über das Buch „Veränderungen in der kapitalistischen Wirtschaft im Gefolge des zweiten Weltkrieges" von E. Varga, a. a. O. 
prozesse des Kapitalismus und leistet einen eigenständigen wertvollen Beitrag für die weitere theoretische Diskussion. Dies bezieht sich sowohl auf seine Thesen zum staatsmonopolistischen Kapitalismus als auch auf die Probleme der Theorie der Lage der Arbeiterklasse wie die Fragen der relativen und absoluten Verelendung des Proletariats oder die Probleme der Arbeiteraristokratie. Gleichzeitig behandelt Eugen Varga wichtige Probleme der Veränderung des kapitalistischen Zyklus nach dem zweiten Weltkrieg und grundlegende Entwicklungstendenzen der kapitalistischen Weltwirtschaft wie die Fragen der Entwicklung des kapitalistischen Marktes, die Agrarkrisen und Fragen des gemeinsamen Marktes. Dabei geht Eugen Varga auch, wie er das in früheren Schriften getan hat, auf eigene Irrtümer und Fehler ein, legt seine neuen Erkenntnisse dar und gibt damit ein Beispiel für die schöpferische Analyse der marxistisch-leninistischen politischen Ökonomie unter den heutigen Bedingungen.

Der dritte Band enthält auch eine umfassende Bibliographie der Arbeiten Eugen Vargas. Sie ist die bisher umfangreichste Erfassung aller Schriften Eugen Vargas und besitzt dadurch einen eigenständigen wissenschaftlichen Wert. Sie wird es den Forschern und Lehrern auf dem Gebiete der politischen Ökonomie des Kapitalismus ermöglichen, sich noch eingehender mit den Schriften Eugen Vargas bekannt zu machen. Dem Akademie-Verlag, besonders seinem Mitarbeiter Rainer Sämisch, gebührt Dank und Anerkennung für die gesamte Förderung dieser dem Jubiläum Eugen Vargas gewidmeten 3bändigen Ausgabe.

Die Herausgeber 
